\title{
DIRICHLET SPACES AND STRONG MARKOV PROCESSES
}

\author{
BY \\ MASATOSHI FUKUSHIMA $\left({ }^{1}\right)$
}

\begin{abstract}
We show that there exists a suitable strong Markov process on the underlying space of each regular Dirichlet space. Potential theoretic concepts due to A. Beurling and J. Deny are then described in terms of the associated strong Markov process. The proof is carried out by developing potential theory for Dirichlet spaces and symmetric Ray processes and by using a method of transformation of underlying spaces.
\end{abstract}

Introduction. This paper is a continuation of [10]. We will use those notions and terminologies adopted in [10].

Let $(X, m, \mathscr{F}, \mathscr{E})$ be a $D$-space. We define $\left(\alpha_{0}-\right)$ capacity of an open set $A \subset X$ by

$$
\begin{aligned}
\operatorname{Cap}(A) & =\inf _{u \in \mathscr{L}_{A}} \mathscr{E}^{\alpha}(u, u) & & \text { if } \mathscr{L}_{A} \neq \varnothing, \\
& =+\infty & & \text { otherwise, }
\end{aligned}
$$

where $\alpha_{0}$ is a fixed positive number and

$$
\mathscr{L}_{A}=\{u \in \mathscr{F} ; u \geqq 1 m \text {-a.e. on } A\} \text {. }
$$

The capacity of an arbitrary set $A \subset X$ is defined by

$$
\operatorname{Cap}(A)=\inf _{A \subset B, B \text { open }} \operatorname{Cap}(B) .
$$

We show in subsection 1.1 that this definition gives us a Choquet capacity $\left({ }^{2}\right)$. A set $A \subset X$ is said to be polar if $A$ has zero capacity. If $A$ is polar, then $m(A)=0$.

From subsection 1.2 to the end of this paper, we will concentrate our attention on regular $D$-spaces. According to Definition 2.3 of [10], a $D$-space is called regular if $m$ is everywhere dense on $X$ and the space $\mathscr{F} \cap C(X)$ is dense both in $\mathscr{F}$ with norm $\mathscr{E}^{\alpha_{0}}$ and in $C(X)$ with uniform norm, $C(X)$ being the space of all continuous functions vanishing at infinity on $X$. Our goal in this paper is to establish the following existence theorem of a strong Markov process.

Received by the editors September 1, 1970.

AMS 1969 subject classifications. Primary 6062; Secondary 3122.

Key words and phrases. Capacity, regular Dirichlet space, quasi-continuity, equilibrium potential, quasi-homeomorphism, Ray process, Hunt process, fine continuity, hitting probability, reference measure.

( $\left.{ }^{1}\right)$ This research was supported by the Center for Advanced Study of the University of Illinois.

$\left({ }^{2}\right)$ This fact has been proved by J. Deny [3] under a kind of regularity condition for a function space. 
THeOREM 4.1. Let $(X, m, \mathscr{F}, \mathscr{E})$ be a regular $D$-space. There exist then a (possibly empty) Borel polar set $B \subset X$ and a right continuous strong Markov process $M=\left(\Omega, \mathscr{M}, \mathscr{M}_{t}, X_{t}, P_{x}\right)$ with state space $X \cup \partial-B$ such that the resolvent of the process $M$ generates the given D-space $\left(\mathscr{F}^{*}, \mathscr{E}\right)\left({ }^{3}\right)$ : if we put

$$
R_{\alpha} f(x)=E_{x}\left(\int_{0}^{+\infty} e^{-\alpha t} f\left(X_{t}\right) d t\right), \quad x \in X-B,
$$

for $f \in L^{2}(X ; m) \cap C(X)$ under the convention that $f(\partial)=0$, then the function $R_{\alpha} f$ belongs to the space $\mathscr{F} *$ and the equation

$$
\mathscr{E}^{\alpha}\left(R_{\alpha} f, v\right)=(f, v)_{X}
$$

holds for every $v \in \mathscr{F}$. Furthermore the state space $X \cup \partial-B$ has no branch point and $M$ is quasi-left continuous on $[0,+\infty)$.

In $\S 4$ we will prove this theorem by constructing these objects $\left(\Omega, \mathscr{M}, \mathscr{M}_{t}, X_{t}, P_{x}\right)$ in a specific way and describing more detailed properties that they possess. It turns out that the process $M$ is actually a Hunt process $\left({ }^{4}\right)$.

Here we give a brief account of our procedure.

$\$ 1$ will provide some basic facts related to a regular $D$-space most of which are well known as the contents of Beurling-Deny's potential theory. We reproduce them because our definition of the regularity is slightly more general than BeurlingDeny's and further our approach to the potential theory is based on the concept of quasi-supermedian functions.

Theorem 2.1 of $\$ 2$ will state that, if two regular $D$-spaces are equivalent in the sense of Definition 4.1 of [10], then their underlying spaces are related by a capacity preserving quasi-homeomorphism $\left({ }^{5}\right)$. We need the regular representation theorem [10] for the proof of Theorem 2.1.

In $\$ 3$ we examine the relationship between two aspects of a strongly regular $D$-space-the potential theoretic one developed in $\$ 1$ and the probability theoretic one corresponding to the associated Ray process. For instance, we prove in Theorem 3.12 that a set $A$ is polar if and only if there is an $m$-negligible Borel set $B \supset A$ such that almost all sample paths of the Ray process starting at any point of $X-B$ will never contact with $B$.

The proof of Theorem 4.1 is accomplished in the following way. Let $(X, m, \mathscr{F}, \mathscr{E})$ be a regular $D$-space. Then by virtue of Theorem 3 of [10], there is a strongly regular $D$-space $(\tilde{X}, \tilde{m}, \tilde{\mathscr{F}}, \mathscr{E})$ which is equivalent to $(X, m, \mathscr{F}, \mathscr{E})$. Owing to Theorem $2.1, X$ is related to $\tilde{X}$ by a capacity preserving quasi-homeomorphism $q$. $q$ will transform the associated Ray process on $\tilde{X}$ into a process on $X$ which turns out to have the properties of Theorem 4.1.

$\left({ }^{3}\right) \mathscr{F}^{*}$ is the quasi-continuous modification of $\mathscr{F}$ (subsection 1.2).

( ${ }^{4}$ See P. A. Meyer [16, Chapitre XVI]. The state space of the process $M$ is not necessarily a locally compact set but a Borel subset of the compactum $X \cup \partial$.

$\left.{ }^{5}\right)$ We can find an analogous reasoning in M. Nakai [17]. 
Thus every regular $D$-space is endowed with a probabilistic structure and we can see that all theorems of $\S 3$ are generalized at once to the case of the regular $D$-space. Subsection 4.2 collects some of the generalizations-an identification of decomposition of the $D$-space and that of an associated Hunt process, an identification of quasi-continuity and q.e. fine continuity, etc. In particular our notion of polar sets turns out to be weaker in general than the usually adopted probabilistic one. They are identical, however, as we will see in subsections 3.6 and 4.2, if and only if the underlying measure $m$ is a reference measure for the process.

Although we go no further at present, it may be asserted that sample paths governed by a Dirichlet space will run along "the roads" indicated by the 0 -order Dirichlet form $\mathscr{E}$ and with "speed" indicated by the underlying measure $m$.

I wish to express my hearty thanks to T. Shiga who read the original version of the manuscript and gave me valuable suggestions.

1. Potential theory for $D$-spaces. Let $(X, m, \mathscr{F}, \mathscr{E})$ be a $D$-space. We do not assume any regularity condition in the first subsection. From subsection 1.2 throughout $\S 1$ we will assume that $(X, m, \mathscr{F}, \mathscr{E})$ is regular.

1.1. Capacity.

THEOREM 1.1. The capacity defined by (0.1) and (0.3) for all subsets of $X$ is a Choquet capacity, that is,

(a) it is increasing,

(b) for any increasing sequence of subsets $A_{n}$ of $X$,

and

$$
\operatorname{Cap}\left(\bigcup_{n} A_{n}\right)=\sup _{n} \operatorname{Cap}\left(A_{n}\right)
$$

(c) for any decreasing sequence of compact subsets $A_{n}$ of $X$,

$$
\operatorname{Cap}\left(\bigcap_{n} A_{n}\right)=\inf _{n} \operatorname{Cap}\left(A_{n}\right)
$$

Furthermore it has the property that

(d) it is nonnegative and countably subadditive.

Our capacity is evidently nonnegative and increasing. Property (c) is also clear. In fact, for any $\varepsilon>0$, there exists an open set $E \supset \bigcap_{n} A_{n}$ such as $\operatorname{Cap}\left(\bigcap_{n} A_{n}\right)$ $\geqq \operatorname{Cap}(E)-\varepsilon$. However, $E \supset A_{n}$ for some $n$ and we have $\operatorname{Cap}\left(\bigcap_{n} \cdot A_{n}\right) \geqq \inf _{n} \operatorname{Cap}\left(A_{n}\right)$.

According to P. A. Meyer [15, III, T23], the other assertions of Theorem 1.1 follow from the next lemma.

LEMMA 1.1. The capacity defined by (0.1) for all open sets of $X$ has the following properties. Denote by $\mathscr{U}$ the class of all open sets $A$ for which $\mathscr{L}_{A} \neq \varnothing$.

(i) It is finite, nonnegative and increasing on $\mathscr{U}$.

(ii) It is strongly subadditive on $\mathscr{U}$ : for any $A, B \in \mathscr{U}$,

$$
\text { Cap }(A \cup B)+\operatorname{Cap}(A \cap B) \leqq \operatorname{Cap}(A)+\operatorname{Cap}(B) .
$$


(iii) If $A_{n} \in \mathscr{U}$ is increasing and $\bigcup_{n} A_{n} \in \mathscr{U}$, then

$$
\operatorname{Cap}\left(\bigcup_{n} A_{n}\right)=\sup _{n} \operatorname{Cap}\left(A_{n}\right) \text {. }
$$

(iv) For any open set $A$ belonging to $\mathscr{U}_{\sigma}$,

$$
\operatorname{Cap}(A)=\sup _{B \subset A, B \in \mathscr{U}} \operatorname{Cap}(B) .
$$

Proof. For $A \in \mathscr{U}$, there exists a unique element $p_{A} \in \mathscr{L}_{A}$ minimizing the quadratic form $\mathscr{E}^{\alpha} \alpha_{0}(u, u)$ in $\mathscr{L}_{A}$, since $\mathscr{L}_{A}$ is a nonempty convex set of $\mathscr{F}$ closed with norm $\mathscr{E} \alpha_{0}$. Evidently,

$$
\operatorname{Cap}(A)=\mathscr{E}^{\alpha_{0}}\left(p_{A}, p_{A}\right) .
$$

Since $\left(0 \vee p_{A}\right) \wedge 1$, being a normal contraction of $p_{A}$, is identical with $p_{A}$, we have

$$
\begin{gathered}
0 \leqq p_{A} \leqq 1 \quad m \text {-a.e. on } X \\
p_{A}=1 \quad m \text {-a.e. on } A .
\end{gathered}
$$

Further we have

$$
\mathscr{E}^{\alpha_{0}}\left(p_{A}, v\right) \geqq 0
$$

for any $v \in \mathscr{F}$ which is nonnegative $m$-a.e. on $A$. This follows from

$$
\mathscr{E}^{\alpha} \alpha_{0}\left(p_{A}+\varepsilon v, p_{A}+\varepsilon v\right) \geqq \mathscr{E}^{\alpha_{0}}\left(p_{A}, p_{A}\right), \quad \varepsilon>0 .
$$

It is easy to see that $p_{A} \in \mathscr{F}$ is characterized by two conditions (1.3) and (1.4). Keeping these in mind, let us prove Lemma 1.1.

(i) Trivial.

(ii) Since $\left|p_{A}-p_{B}\right|$ is a normal contraction of $p_{A}-p_{B}$, we have

$$
\mathscr{E}^{\alpha_{0}}\left(p_{A} \vee p_{B}, p_{A} \vee p_{B}\right)+\mathscr{E}^{\alpha_{0}}\left(p_{A} \wedge p_{B}, p_{A} \wedge p_{B}\right) \leqq \mathscr{E}^{\alpha_{0}}\left(p_{A}, p_{A}\right)+\mathscr{E}^{\alpha_{0}}\left(p_{B}, p_{B}\right),
$$

which implies the desired inequality.

(iii) For $n>m$,

$$
\mathscr{E}_{0} 0\left(p_{A_{n}}-p_{A_{m}}, p_{A_{n}}-p_{A_{m}}\right)=\operatorname{Cap}\left(A_{n}\right)-\operatorname{Cap}\left(A_{m}\right) .
$$

Since Cap $\left(A_{n}\right)$ is bounded from above (by the capacity of $A=\cup_{n} A_{n}$ ), the preceding equality means that $p_{A_{n}}$ converges to a $u_{0} \in \mathscr{F}$ in norm $\mathscr{E} \alpha_{0} . u_{0}=1 \mathrm{~m}$-a.e. on $A$ because $p_{A_{n}}=1 m$-a.e. on $A_{n}$. Moreover $\mathscr{E}^{\alpha_{0}}\left(u_{0}, v\right)=\lim _{n \rightarrow+\infty} \mathscr{E} \alpha_{0}\left(p_{A_{n}}, v\right) \geqq 0$ for every $v \in \mathscr{F}$ which is nonnegative $m$-a.e. on $A$. Thus $u_{0}=p_{A}$ and

$$
\lim _{n \rightarrow+\infty} \operatorname{Cap}\left(A_{n}\right)=\lim _{n \rightarrow+\infty} \mathscr{E}^{\alpha_{0}}\left(p_{A_{n}}, p_{A_{n}}\right)=\mathscr{E}^{\alpha} \alpha_{0}\left(p_{A}, p_{A}\right)=\operatorname{Cap}(A) .
$$

(iv) Consider an element $A$ of $\mathscr{U}_{\sigma}$ and put $c=\sup _{B \subset A, B \in \mathscr{U}} \operatorname{Cap}(B)$. There exists an increasing sequence of open sets $A_{n} \in \mathscr{U}$ such that $\bigcup_{n} A_{n}=A$. By making use of statement (iii), we easily obtain the equality $c=\lim _{n \rightarrow+\infty} \operatorname{Cap}\left(A_{n}\right) \leqq+\infty$. Now this equality combined with exactly the same argument as in the proof of (iii) leads us to 
the conclusion that $c$ is finite if and only if $A \in \mathscr{U}$ and in this case $c=\operatorname{Cap}(A)$. Hence, if $c=+\infty$, then $A \notin \mathscr{U}$ and $\operatorname{Cap}(A)=+\infty$ by definition. In any case, we get the desired equality. The proof of Lemma 1.1 is complete.

Theorem 1.1 combined with Choquet's theorem implies that, for any analytic set $A \subset X$,

$$
\operatorname{Cap}(A)=\sup _{K \subset A, K \text { compact }} \operatorname{Cap}(K) .
$$

In subsection 1.5 , we will give some characterizations of the capacity for compact sets in the case of the regular $D$-space.

A subset $A$ of $X$ is called polar if Cap $(A)=0$. The expression "quasi-everywhere" or "q.e." means "except for a polar set". Let $E$ be an open set of $X$. A function $u$ defined q.e. on $E$ is called quasi-continuous on $E$ if, for any $\varepsilon>0$, there exists an open set $\omega \subset E$ such that Cap $(\omega)<\varepsilon$ and the restriction of $u$ to $X-\omega$ is continuous there. Quasi-continuous functions on $X$ are simply said to be quasi-continuous.

THEOREM 1.2. (i) If $A$ is polar, then $m(A)=0$.

(ii) If $u_{1}$ and $u_{2}$ are quasi-continuous on an open set $E \subset X$ and $u_{1} \geqq u_{2} m$-a.e. on $E$, then $u_{1} \geqq u_{2}$ q.e. on $E$.

Proof. (i) This is evident in view of the inequality Cap $(A) \geqq \alpha_{0} m(A)$ for the open set $A$, which is immediate from (0.1).

(ii) Fix an $\varepsilon>0$. There exists then an open set $\omega \subset E$ with Cap $(\omega)<\varepsilon$ such that $u_{1}$ and $u_{2}$ are continuous on $E-\omega$. Put $\omega^{\prime}=\{x \in E$; there exists a neighborhood $U(x)$ of $x$ such that $U(x) \subset E$ and $m(U(x)-\omega)=0\}$. It is easy to see that $\omega^{\prime}$ is an open set, $\omega \subset \omega^{\prime} \subset E$ and $m\left(\omega^{\prime}-\omega\right)=0\left({ }^{6}\right)$. Hence $\mathscr{L} \omega^{\prime}=\mathscr{L} \omega$ and, by $(0.1)$, Cap $\left(\omega^{\prime}\right)$ $=\mathrm{Cap}(\omega)<\varepsilon$. Now let us show $A \subset \omega^{\prime}$, where $A=\left\{x \in E ; u_{1}(x)<u_{2}(x)\right\}$. Suppose that there is an element $x \in A \cap\left(E-\omega^{\prime}\right)$. Since $x \in A \cap(E-\omega)$, there exists a $U(x) \subset E$ such that $u_{1}<u_{2}$ on $U(x)-\omega$. However, $m(U(x)-\omega) \neq 0$ because $x \in E-\omega^{\prime}$. This contradicts the assumption that $u_{1} \geqq u_{2} m$-a.e. on $E$. Thus $A \subset \omega^{\prime}$ and Cap $(A)<\varepsilon$, proving that $A$ is polar.

1.2. Quasi-continuous modification $\mathscr{F}^{*}$. From now on we assume that the given $D$-space $(X, m, \mathscr{F}, \mathscr{E})$ is regular.

Theorem 1.2(ii) then implies that Cap $(A)>0$ for every nonempty open set $A$. Moreover, if a subset $A \subset X$ has a compact closure, then Cap $(A)$ is finite. In fact, $A$ is then included in an open set $E$ with compact closure. $\mathscr{L}_{E}$ is not empty for such an $E$.

THeOREM 1.3. For any $u \in \mathscr{F}$, there exists $u_{n} \in \mathscr{F} \cap C(X)$ and increasing closed subsets $F_{m}$ such that $\mathscr{E}_{0}\left(u_{n}-u, u_{n}-u\right) \rightarrow 0$, Cap $\left(\bigcap_{m=1}^{\infty} F_{m}^{c}\right)=0$ and $u_{n}$ converges

${ }^{(6)}$ Since $X$ is assumed to be separable, we can use the Lindelöf covering theorem to prove this point. Cf. Hilfssatz 5.9 in C. Constantinescu and A. Cornea, Ideal ränder Riemannscher flächen, Springer, 1963. 
uniformly on each $F_{m}$. The limit function $u^{*}$ of $u_{n}$ is quasi-continuous and equal to $u$ m-a.e.

Proof. By means of $(0.1)$, we have

$$
\operatorname{Cap}\{x ;|v(x)|>\varepsilon\} \leqq \mathscr{E}^{\alpha_{0}}(v, v) / \varepsilon^{2}
$$

for any $\varepsilon>0$ and $v \in \mathscr{F} \cap C(X)$. Take $u \in \mathscr{F}$ and find $u_{n} \in \mathscr{F} \cap C(X)$ converging to $u$ with $\mathscr{E}^{\alpha_{0}}$-norm. Subtracting a suitable subsequence if necessary, we can assume that $\operatorname{Cap}\left(G_{n}\right) \leqq 1 / 2^{n}$ for the open set $G_{n}=\left\{x ;\left|u_{n}(x)-u_{n+1}(x)\right|>1 / 2^{n}\right\}$. The statement of Theorem 1.3 holds for $F_{m}=\bigcap_{n=m}^{+\infty} G_{n}^{c}$.

If a function $u$ is defined $m$-a.e. on $X$ and if $u^{*}$ is quasi-continuous and equal to $u$ $m$-a.e. on $X$, then $u^{*}$ is called a quasi-continuous modification of $u$. Denote by $\mathscr{F} *$ the set of all quasi-continuous modifications of functions of $\mathscr{F}$. We regard two functions of $\mathscr{F}^{*}$ to be equivalent if they are identical q.e. on $X$. On account of Theorems 1.2 and 1.3, the equivalence classes of $\mathscr{F}^{*}$ with inner product $\mathscr{E}^{\alpha}$ form a real Hilbert space which is just identical with the space $(\mathscr{F}, \mathscr{E} \alpha)$, two functions of $\mathscr{F}$ being identified if they coincide $m$-a.e.

The next lemma can be proved exactly in the same manner as in J. Deny and J. Lions [5, II, Lemme 4.1 and Théorème 4.1].

LEMMA 1.2. (i) The estimate (1.6) holds for any $\varepsilon>0$ and $v \in \mathscr{F *}$.

(ii) If $u_{n}$ is a Cauchy sequence in $\left(\mathscr{F}^{*}, \mathscr{E}^{\alpha}\right)$, then $u_{n}$ converges to a function $u \in \mathscr{F}^{*}$ with $\mathscr{E} \alpha_{0-n o r m}$. Further there exists a subsequence $n_{k}$ such that $\lim _{n_{k} \rightarrow+\infty} u_{n_{k}}(x)$ $=u(x)$ q.e. on $X$.

1.3. Quasi-supermedian functions and potentials. Let $\left\{G_{\alpha}, \alpha>0\right\}$ be the $L^{2}-$ resolvent associated with the $D$-space $(\mathscr{F}, \mathscr{E})$. Each $G_{\alpha}$ is a linear operator from $L^{2}(X ; m)$ into $\mathscr{F}$. From now on, however, we regard $G_{\alpha}$ as a linear operator from $L^{2}(X ; m)$ into the space $\mathscr{F}^{*}$, as the preceding subsection 1.2 admits us to do.

We call a function $u \in L^{2}(X ; m)\left(\alpha_{0}-\right)$ quasi-supermedian if the following two conditions are satisfied.

(1.7) $u$ is quasi-continuous and $u \geqq 0$ q.e.

(1.8) $\beta G_{\beta+\alpha_{0}} u \leqq u$ q.e., $\beta>0$.

LEMMA 1.3. A function $u \in \mathscr{F}^{*}$ is quasi-supermedian if and only if $\mathscr{E}^{\alpha} 0(u, v) \geqq 0$ for every $v \in \mathscr{F}^{*}$ such that $v \geqq 0$ q.e.

Proof. If $u \in \mathscr{F}^{*}$ is quasi-supermedian then according to Lemma 2.1 of [10],

$$
\mathscr{E}^{\alpha} \alpha_{0}(u, v)=\lim _{\beta \rightarrow+\infty} \beta\left(u-\beta G_{\beta+\alpha_{0}} u, v\right)_{X} \geqq 0 \quad \text { for } v \in \mathscr{F}
$$

such that $v \geqq 0 m$-a.e.

Conversely assume that $u \in \mathscr{F} *$ satisfies the inequality $\mathscr{E}^{\alpha_{0}}(u, v) \geqq 0$ for every $v \in \mathscr{F}^{*}$ which is nonnegative q.e. on $X$. Then $u$ is the unique element minimizing the norm $\mathscr{E}^{\alpha_{o}}(w, w)$ in the convex set $\mathscr{L}_{u}=\left\{w \in \mathscr{F}^{*} ; w \geqq u\right.$ q.e. $\} .|u|$ is a normal 
contraction of $u$ and belongs to $\mathscr{L}_{u}$. Thus $u=|u| \geqq 0$ q.e. proving (1.7). Furthermore we have, for any $v \in L^{2}(X ; m)$ such as $v \geqq 0 m$-a.e.,

$$
\left(u-\beta G_{\beta+\alpha_{0}} u, v\right)_{X}=\mathscr{E}^{\beta+\alpha_{0}}\left(u, G_{\beta+\alpha_{0}} u\right)-\beta\left(u, G_{\beta+\alpha_{0}} v\right)_{X}=\mathscr{E}_{0} \alpha_{0}\left(u, G_{\beta+\alpha_{0}} v\right)
$$

which is nonnegative because $G_{\beta+\alpha_{0}} v \in \mathscr{F}^{*}$ and $G_{\beta+\alpha_{0}} v \geqq 0$ q.e. (Theorem 1.2(ii)). This proves (1.8). The proof of Lemma 1.3 is complete.

Denote by $M_{0}^{+}$the set of all nonnegative Borel measures $\mu$ on $X$ satisfying the following two conditions:

$$
\mathscr{F} \cap C(X) \subset L^{1}(X ; \mu)
$$

(1.10) There exists a function $u \in \mathscr{F} *$ such that

$$
\mathscr{E}^{\alpha_{0}}(u, v)=\int_{X} v(x) \mu(d x) \text { for any } v \in \mathscr{F} \cap C(X) .
$$

The function $u$ of (1.10) is uniquely determined by $\mu \in M_{0}^{+}$. It is called the $\left(\alpha_{0^{-}}\right)$ potential of $\mu$ and denoted by $U \mu$.

Every $\mu \in M_{0}^{+}$is a Radon measure on $X$, namely, $\mu$ is finite for any compactum.

Any $u \in \mathscr{F}^{*}$ defines a linear functional $l_{u}$ on $\mathscr{F} \cap C(X)$ by $l_{u}(v)=\mathscr{E}^{\alpha} \alpha_{o}(u, v)$, $v \in \mathscr{F} \cap C(X)$. Meanwhile, $\mathscr{F} \cap C(X)$ is closed under lattice operations and $v \wedge 1 \in \mathscr{F} \cap C(X)$ for any $v \in \mathscr{F} \cap C(X)$ [10, Lemma 4.1]. Therefore by the general theory of Daniell integral [14, Chapter 3], $l_{u}$ is an integral by means of the Baire measure with respect to the class $\mathscr{F} \cap C(X)$ if and only if $l_{u}$ is a positive functional and continuous under monotone limits. Since the Baire family generated by $\mathscr{F} \cap C(X)$ is the set of Borel functions, we get the following

LEMMA 1.4. $u \in \mathscr{F}^{*}$ is a potential if and only if

(1.11) $\mathscr{E} \alpha_{0}(u, v) \geqq 0$ for any nonnegative $v \in \mathscr{F} \cap C(X)$.

(1.12) $\mathscr{E}^{\alpha_{0}}\left(u, v_{n}\right) \downarrow 0$ if $v_{n} \in \mathscr{F} \cap C(X)$ converges monotonically to zero.

When $X$ is compact, condition (1.12) is superfluous. If $u$ is a potential, $u$ determines the associated measure $\mu \in M_{0}^{+}$uniquely.

Now we will state the relation of quasi-supermedian functions and potentials.

THEOREM 1.4. A function $u \in \mathscr{F}^{*}$ is a potential if and only if $u$ is a quasisupermedian function satisfying condition (1.12).

Proof. It suffices to show that condition (1.11) implies the stronger condition of Lemma 1.3. Suppose that $u \in \mathscr{F}^{*}$ satisfies (1.11). Let $v \in \mathscr{F} *$ be nonnegative q.e. and $v_{n} \in \mathscr{F} \cap C(X)$ be a sequence converging to $v$ in $\mathscr{E} \alpha_{0}$-norm. Consider $v_{n}^{+}$ $=v_{n} \vee 0 \in \mathscr{F} \cap C(X)$. Then $\mathscr{E}^{\alpha} \alpha_{0}\left(v_{n}^{+}, v_{n}^{+}\right) \leqq \mathscr{E}^{\alpha} \alpha_{0}\left(v_{n}, v_{n}\right)$ is bounded in $n$ and

$$
\mathscr{E}^{\alpha_{0}}\left(v_{n}^{+}, G_{\alpha_{0}} f\right)=\left(v_{n}^{+}, f\right)_{X} \rightarrow(v, f)_{X}=\mathscr{E}^{\alpha_{0}}\left(v, G_{\alpha_{v}} f\right) \text { for any } f \in L^{2}(X ; m) \text {. }
$$

Since $G_{\alpha_{0}}\left(L^{2}\right)$ is dense in $\mathscr{F}, v_{n}^{+}$converges to $v$ weakly. In particular,

$$
\mathscr{E}^{\alpha} \alpha_{0}(u, v)=\lim _{n \rightarrow+\infty} \mathscr{E}^{\alpha} \alpha_{0}\left(u, v_{n}^{+}\right) \geqq 0 .
$$


1.4. Basic properties of potentials. Define the support $S \mu$ of $\mu \in M_{0}^{+}$by $S \mu$ $=\left\{x \in X ; \mu\left(U_{x}\right) \neq 0\right.$ for any neighborhood $U_{x}$ of $\left.x\right\} . S \mu$ is a closed set. Let us begin with an approximation lemma.

Lemma 1.5. Suppose that $\mu \in M_{0}^{+}$has a compact support $S \mu$. Then for any open set $E$ such that $E \supset S \mu$ and $\bar{E}$ is compact, there exist nonnegative functions $f_{n} \in L^{2}(X ; m)$ which vanish $m$-a.e. on $X-E$ and satisfy

(1.13) $f_{n} \cdot m \rightarrow \mu$ vaguely as measures,

(1.14) $G_{\alpha_{0}} f_{n} \rightarrow U \mu$ weakly in $\left(\mathscr{F}, \mathscr{E} \alpha_{0}\right)$.

Proof. By virtue of Theorem 1.4, the potential $U \mu$ is quasi-supermedian, and so

$$
g_{\beta}=\beta\left(U \mu-\beta G_{\beta+\alpha_{0}}(U \mu)\right)
$$

is nonnegative. Let us prove the equality

$$
\lim _{\beta \rightarrow+\infty} \int_{X} v(x) g_{\beta}(x) m(d x)=\int_{K} v(x) \mu(d x)
$$

for every continuous function $v$ such as $|v| \leqq v_{0}$, where $K=S \mu$ and $v_{0}$ is an arbitrarily fixed function in $\mathscr{F} \cap C(X)$. According to Lemma 2.1 of [10] and (1.10), the equality (1.16) is true for every $v \in \mathscr{F} \cap C(X)$. Incidentally the measures $g_{\beta} \cdot m$ are uniformly bounded in $\beta$ on any compactum. Turning to the case of general $v$, choose $v_{k} \in \mathscr{F} \cap C(X)$ such as $\left|v_{k}\right| \leqq v_{0}$ and $\left\|v_{k}-v\right\|_{\infty} \rightarrow 0, k \rightarrow+\infty$, and observe the following inequality:

$$
\begin{aligned}
\mid \int_{X} v(x) g_{\beta}(x) m(d x) & -\int_{K} v(x) \mu(d x) \mid \\
\leqq & \left|\int_{X} v_{k}(x) g_{\beta}(x) m(d x)-\int_{K} v_{k}(x) \mu(d x)\right|+\int_{K}\left|v(x)-v_{k}(x)\right| \mu(d x) \\
& +\int_{F}\left|v(x)-v_{k}(x)\right| g_{\beta}(x) m(d x)+2 \int_{X-F} v_{0}(x) g_{\beta}(x) m(d x) .
\end{aligned}
$$

For any $\varepsilon>0$, take a compactum $F$ such that $v_{0}<\varepsilon$ on $X-F$, then the superior limit in $\beta$ of the last term of the right-hand side is less than $2 \int_{K}\left(v_{0} \wedge \varepsilon\right) \mu(d x) \leqq 2 \varepsilon \cdot \mu(K)$. Now by taking sufficiently large $k$, we can make the superior limit in $\beta$ of the right-hand side arbitrarily small.

It is clear that (1.16) implies (1.13) with

$$
f_{n}(x)=g_{n}(x) \chi_{E}(x), \quad x \in X,
$$

$\chi_{E}$ being the indicator function of the open set $E$. It follows from (1.13) that

$$
\mathscr{E}^{\alpha_{0}}\left(G_{\alpha_{0}} f_{n}, v\right)=\left(f_{n}, v\right)_{X} \rightarrow \int_{K} v(x) \mu(d x)=\mathscr{E}^{\alpha_{0}}(U \mu, v)
$$

for $v \in \mathscr{F} \cap C(X)$. 
Since

$$
\begin{aligned}
\mathscr{E}^{\alpha_{0}}\left(G_{\alpha_{0}} f_{n}, G_{\alpha_{0}} f_{n}\right) & =\left(f_{n}, G_{\alpha_{0}} f_{n}\right)_{X} \leqq\left(g_{n}, G_{\alpha_{0}} g_{n}\right)_{X} \\
& =\left(g_{n}, n G_{n+\alpha_{0}} U \mu\right)_{X} \leqq\left(g_{n}, U \mu\right)_{X}
\end{aligned}
$$

is uniformly bounded by $\mathscr{E}_{0}(U \mu, U \mu)$, we arrive at (1.14).

We will point out here that, for any $\mu \in M_{0}^{+}$and compactum $K$, the measure $\mu_{K}$ defined by $\mu_{K}(\cdot)=\mu(K \cap \cdot)$ is also in $M_{0}^{+}$and

$$
\mathscr{E}^{\alpha_{0}}\left(U \mu_{K}, U \mu_{K}\right) \leqq \mathscr{E}^{\alpha} \alpha_{0}(U \mu, U \mu) .
$$

Indeed, the inequality

$$
\begin{aligned}
\left|\int_{X} v(x) \mu_{K}(d x)\right| & \leqq \int_{X}|v(x)| \mu(d x)=\mathscr{E}^{\alpha_{0}}(U \mu,|v|) \\
& \leqq \sqrt{ }\left(\mathscr{E}^{\alpha_{0}}(U \mu, U \mu)\right) \sqrt{ }\left(\mathscr{E}^{\alpha} \alpha_{0}(v, v)\right), \quad v \in \mathscr{F} \cap C(X),
\end{aligned}
$$

implies the existence of the potential $U \mu_{K} \in \mathscr{F}$ satisfying (1.10). Further we have $\mu-\mu_{K} \in M_{0}^{+}$. Therefore, by means of Lemma 1.3 and Theorem 1.4,

$$
\mathscr{E} \alpha_{0}\left(U\left(\mu-\mu_{K}\right), U \mu_{K}\right) \geqq 0,
$$

which means (1.18). Keeping this in mind, let us proceed to

THEOREM $1.5\left(^{7}\right)$. (i) If $A$ is polar, then $\mu(A)=0$ for every $\mu \in M_{0}^{+}$.

(ii) If $\mu \in M_{0}^{+}$, then $\mathscr{F}^{*} \subset L^{1}(X ; \mu)$ and

$$
\mathscr{E} \alpha_{0}(U \mu, u)=\int_{X} u(x) \mu(d x), \quad u \in \mathscr{F} *
$$

Proof. (i) Suppose that $A$ is polar and $\mu \in M_{0}^{+}$. For any $\varepsilon>0$, there is an open set $E \supset A$ with Cap $(E)<\varepsilon$. Take any compactum $K$ included in $E$ and choose $f_{n}$ satisfying conditions of the preceding lemma for $\mu_{K}$ and an open set $E_{1} \subset E$ with compact closure $\bar{E}_{1}$. Then,

$$
\mathscr{E}^{\alpha_{0}}\left(U \mu_{K}, p_{E}\right)=\lim _{n \rightarrow+\infty} \mathscr{E}^{\alpha_{0}}\left(G_{\alpha_{0}} f_{n}, p_{E}\right)=\lim _{n \rightarrow+\infty} \int_{E_{1}} f_{n}(x) m(d x)=\mu(K) .
$$

Hence

$$
\mu(K) \leqq \sqrt{ }\left(\mathscr{E}^{\alpha_{o}}\left(U \mu_{K}, U \mu_{K}\right)\right) \sqrt{ }\left(\mathscr{E}^{\alpha_{0}}\left(p_{E}, p_{E}\right)\right) \leqq \sqrt{ }\left(\mathscr{E}^{\alpha_{o}}(U \mu, U \mu)\right) \cdot \sqrt{ }(\operatorname{Cap}(E))
$$

and

$$
\mu(E) \leqq \sqrt{ } \varepsilon \cdot \sqrt{ }\left(\mathscr{E}^{\alpha_{0}}(U \mu, U \mu)\right)
$$

Thus $\mu(A)=0$.

${ }^{(7)}$ This is a version of Theorem 4(iii) of [1] whose proof was recently given in [4]. 
(ii) Consider $\mu \in M_{0}^{+}$and $u \in \mathscr{F} *$. It is clear from assertion (i) that $u$ is $\mu$ measurable. Let $u_{n} \in \mathscr{F} \cap C(X), F_{k} \subset X$ and $u^{*}$ be those of Theorem 1.3 for the present $u$. It suffices to show that $u^{*} \in L^{1}(X ; \mu)$ and

$$
\mathscr{E}^{\alpha_{0}}(U \mu, u)=\int_{X} u^{*}(x) \mu(d x)
$$

because $u=u^{*}$ q.e. and the right-hand sides of (1.19) and (1.20) are identical.

Let us prove (1.20). We may assume that each $F_{k}$ is compact. Consider the sequence of measures $\mu_{k}=\mu_{F_{k}}$, then

$$
\begin{aligned}
\mathscr{E}^{\alpha_{0}}\left(U \mu_{k}, v\right) & =\int_{F_{k}} v(x) \mu(d x) \underset{k \rightarrow+\infty}{\longrightarrow} \int_{\bigcup_{k} F_{k}} v(x) \mu(d x) \\
& =\int_{X} v(x) \mu(d x)=\mathscr{E}^{\alpha} \alpha_{0}(U \mu, v), \quad v \in \mathscr{F} \cap C(X),
\end{aligned}
$$

which, combined with (1.18), implies that $U \mu_{k}$ converges to $U \mu$ weakly in $\left(\mathscr{F}, \mathscr{E}^{\alpha} \alpha_{0}\right)$. On the other hand,

$$
\begin{aligned}
\mathscr{E}_{0}\left(U \mu_{k}, u\right) & =\lim _{n \rightarrow+\infty} \mathscr{E}_{0}\left(U \mu_{k}, u_{n}\right) \\
& =\lim _{n \rightarrow+\infty} \int_{F_{k}} u_{n}(x) \mu(d x)=\int_{F_{k}} u^{*}(x) \mu(d x) .
\end{aligned}
$$

Therefore,

$$
\mathscr{E}^{\alpha_{0}}(U \mu, u)=\lim _{k \rightarrow+\infty} \mathscr{E}^{\alpha_{0}}\left(U \mu_{k}, u\right)=\int_{\bigcup_{k F_{k}}} u^{*}(x) \mu(d x)=\int_{X} u^{*}(x) \mu(d x)
$$

THEOREM 1.6. Let $K$ be a compact set. Then, for $u \in \mathscr{F}^{*}$, the next three conditions are mutually equivalent:

(i) $u$ is a potential $U \mu$ with $S \mu \subset K$.

(ii) $\mathscr{E}^{\alpha} 0(u, v) \geqq 0$ for any $v \in \mathscr{F} \cap C(X)$ which is nonnegative on $K$.

(iii) $\mathscr{E}^{\alpha} 0(u, v) \geqq 0$ for any $v \in \mathscr{F}^{*}$ which is nonnegative q.e. on $K$.

Proof. Owing to Theorem 1.5, (i) implies (iii). Trivially, (iii) implies (ii). All we have to do is to derive (i) from (ii). Suppose that $u \in \mathscr{F}^{*}$ satisfies condition (ii). We will first prove that $u$ has the properties (1.11) and (1.12). (1.11) is trivial. Let $w$ be a function of $\mathscr{F} \cap C(X)$ which is no less than 1 on $K$. If $v_{n} \in \mathscr{F} \cap C(X)$ is decreasing to zero, then $v_{n}$ converges uniformly on $X$ and $a_{n}=\sup _{x \in K} v_{n}(x)$ decreases to zero. Since $v_{n} \leqq a_{n} w$ on $K$,

$$
\mathscr{E}^{\alpha_{0}}\left(u, v_{n}\right) \leqq a_{n} \mathscr{E}^{\alpha} 0(u, w) \rightarrow 0, \quad n \rightarrow+\infty .
$$

Thus, $u$ satisfies (1.12). By means of Lemma $1.4, u$ is a potential of a measure $\mu \in M_{0}^{+}$. In view of equation (1.10) and condition (ii), we have $S \mu \subset K$.

1.5. Equilibrium potential and capacity for the compact set. We will first define equilibrium potentials for open sets in the class $\mathscr{U}$ of the subsection 1.1 and study 
their properties. Let $A$ be in $\mathscr{U}$ and $p_{A}$ be the function of $\mathscr{F}$ which is characterized by (1.3) and (1.4). Denote by $e_{A}$ any quasi-continuous modification of $p_{A}$. We call $e_{A}$ the $\left(\alpha_{0^{-}}\right)$equilibrium potential for the open set $A \in \mathscr{U}$. According to Theorem 1.2, $e_{A}$ has the following properties:

(1.21) $\operatorname{Cap}(A)=\mathscr{E} \alpha_{0}\left(e_{A}, e_{A}\right)$.

(1.22) $e_{A}=1$ q.e. on $A$.

(1.23) $\mathscr{E}^{\alpha} \alpha_{0}\left(e_{A}, v\right) \geqq 0$ for any $v \in \mathscr{F}^{*}$ which is nonnegative q.e. on $A$.

$e_{A} \in \mathscr{F}^{*}$ is characterized by (1.22) and (1.23) and indeed, it is a unique element which minimizes the norm $\mathscr{E}^{\alpha} 0(u, u)$ in the convex set $\{u \in \mathscr{F} * ; u \geqq 1$ q.e. on $A\}$ of $\mathscr{F} *$. Obviously $e_{A}$ is a quasi-supermedian function.

In the particular case when the closure $\bar{A}$ of $A$ is compact, we can see by Theorem 1.6 and (1.23) that $e_{A}$ is a potential of a measure $\nu_{A} \in M_{0}^{+}$with $S_{v_{A}} \subset \bar{A}$. We call $\nu_{A}$ the equilibrium distribution for the open set $A$. We have

$$
\operatorname{Cap}(A)=\nu_{A}(\bar{A})
$$

because there is a function $w \in \mathscr{F} \cap C(X)$ which is equal to 1 on $\bar{A}$ and we get $\operatorname{Cap}(A)=\mathscr{E}^{\alpha_{0}}\left(e_{A}, w\right)=\nu_{A}(\bar{A})$.

Now consider any compact set $K$ of $X$ and put $\mathscr{L}_{K}^{*}=\left\{u \in \mathscr{F}^{*} ; u \geqq 1\right.$ q.e. on $\left.K\right\}$. $\mathscr{L}_{K}^{*}$ is a nonempty convex set of $\mathscr{F}^{*}$ and closed in norm $\mathscr{E}^{\alpha_{0}}$ according to Lemma 1.2. Therefore there is a unique element $e_{K}$ of $\mathscr{L}_{K}^{*}$ which minimizes the quadratic form $\mathscr{E}^{\alpha_{0}}(u, u)$ in $\mathscr{L}_{K}^{*}$. We call $e_{K}$ the $\left(\alpha_{0^{-}}\right)$equilibrium potential for the compactum $K$. It is easy to see that $e_{K}$ is characterized as an element of $\mathscr{F} *$ which has the following two properties:

(1.25) $e_{K}=1$ q.e. on $K$.

(1.26) $\mathscr{E}^{\alpha}{ }_{0}\left(e_{K}, v\right) \geqq 0$ for any $v \in \mathscr{F}^{*}$ which is nonnegative q.e. on $K$.

By virtue of Theorem 1.6 and (1.26), we see that $e_{K}$ is a potential of a measure $\nu_{K} \in M_{0}^{+}$with $S \nu_{K} \subset K$. We call $\nu_{K}$ the equilibrium distribution for the compactum $K$.

THEOREM 1.7. Let $K$ be compact.

(i) The equilibrium potential $e_{K}$ is characterized as an element of $\mathscr{F}^{*}$ possessing properties (1.25) and

$$
\mathscr{E}^{\alpha_{0}}\left(e_{K}, v\right) \geqq 0 \text { for any } v \in \mathscr{F} \cap C(X)
$$

which is nonnegative on $K$.

(ii) The next equalities hold:

$$
\begin{aligned}
& \operatorname{Cap}(K)=\mathscr{E}^{\alpha_{0}}\left(e_{K}, e_{K}\right)=\nu_{K}(K) . \\
& \operatorname{Cap}(K)=\inf _{u \in \mathscr{C}_{K}} \mathscr{E}^{\alpha_{0}}(u, u),
\end{aligned}
$$

where $\mathscr{C}_{K}=\{u \in \mathscr{F} \cap C(X) ; u \geqq 1$ on $K\}$.

Proof. (i) is evident, since (1.27) is equivalent to (1.26) by virtue of Theorem 1.6. The second equality of (1.28) is immediate from (1.19) and (1.25). 
Let us prove the first equality of (1.28). For any $\varepsilon>0$, there is an open set $A \supset K$ such that $\operatorname{Cap}(K)+\varepsilon>\operatorname{Cap}(A) . A$ is in $\mathscr{U}$. By (1.21), (1.22), (1.25) and (1.26), we have $\mathscr{E}^{\alpha} \alpha_{0}\left(e_{A}, e_{K}\right)=\mathscr{E}^{\alpha_{0}}\left(e_{K}, e_{K}\right)$ and $0 \leqq \mathscr{E}^{\alpha_{0}}\left(e_{A}-e_{K}, e_{A}-e_{K}\right)=\operatorname{Cap}(A)-\mathscr{E}^{\alpha_{0}}\left(e_{K}, e_{K}\right)$. Hence we get the inequality $\operatorname{Cap}(K) \geqq \mathscr{E}^{\alpha} \alpha_{0}\left(e_{K}, e_{K}\right)$. In order to obtain the converse inequality, let us take a sequence of open sets $A_{n}$ such that $\bar{A}_{n}$ is compact, $A_{n} \supset \bar{A}_{n+1}$ and $\bigcap_{n=1}^{\infty} A_{n}=K$. Let $e_{n}$ and $\nu_{n}$ be the equilibrium potential and distribution for $A_{n}$ respectively. Since $\mathscr{E}^{\alpha_{0}}\left(e_{n}-e_{m}, e_{n}-e_{m}\right)=\operatorname{Cap}\left(A_{n}\right)-\operatorname{Cap}\left(A_{m}\right), n<m, e_{n}$ converges to some $e_{0} \in \mathscr{F}^{*}$ in $\mathscr{E}^{\alpha_{0}}$-norm. Since $e_{n}=1$ q.e. on $A_{n}, e_{0}$ has the property (1.25). On the other hand, $\nu_{n}$ concentrates on $\bar{A}_{n}$ and $\nu_{n}\left(\bar{A}_{n}\right)=\operatorname{Cap}\left(A_{n}\right) \leqq \operatorname{Cap}\left(A_{1}\right)$ by (1.24). Therefore a subsequence of $\nu_{n}$ converges weakly to a measure $\nu_{0}$ whose support is in $K$. Now the equality $\mathscr{E}^{\alpha_{0}}\left(e_{n}, v\right)=\int_{A_{n}} v(x) \nu_{n}(d x)$ leads us to $\mathscr{E}^{\alpha} 0\left(e_{0}, v\right)$ $=\int_{K} v(x) \nu_{0}(d x), v \in \mathscr{F} \cap C(X)$, which enables us to conclude that $e_{0}$ has the property (1.27). Thus, by statement (i), we see that $e_{0}=e_{K}$ and $\mathscr{E}^{\alpha_{0}}\left(e_{K}, e_{K}\right)$ $=\lim _{n \rightarrow+\infty} \mathscr{E}^{\alpha_{0}}\left(e_{n}, e_{n}\right)=\lim _{n \rightarrow+\infty} \operatorname{Cap}\left(A_{n}\right) \geqq \operatorname{Cap}(K)$.

Finally, we will show the equality (1.29). Put $c=\inf _{u \in \mathscr{C}_{K}} \mathscr{E} \alpha_{0}(u, u)$ and take a minimizing sequence $u_{n} \in \mathscr{C}_{K}: \lim _{n \rightarrow+\infty} \mathscr{E} \alpha_{0}\left(u_{n}, u_{n}\right)=c$. It is easy to see that $u_{n}$ then forms a Cauchy sequence in norm $\mathscr{E}^{\alpha_{0}}$ and the limit function $u_{0} \in \mathscr{F} *$ does not depend on the choice of the minimizing sequence $u_{n}$. Since $u_{n} \wedge 1 \in \mathscr{C}_{K}$ forms a minimizing sequence as well, we have $u_{0}=1$ q.e. on $K$ according to Lemma 1.2. Further the property (1.27) for $u_{0}$ can be derived from the inequality

$$
\mathscr{E}^{\alpha} \alpha_{0}\left(u_{n}+\varepsilon v, u_{n}+\varepsilon v\right) \geqq \mathscr{E}^{\alpha} 0\left(u_{0}, u_{0}\right)
$$

which holds for any $\varepsilon>0$ and $v \in \mathscr{F} \cap C(X)$ such as $v \geqq 0$ on $K$. Therefore, statement (i) means that $u_{0}=e_{K}$ and $c=\mathscr{E}^{\alpha_{0}}\left(u_{0}, u_{0}\right)=\operatorname{Cap}(K)$. The proof of Theorem 1.7 is complete.

2. Transformation of underlying spaces. Consider two regular $D$-spaces $(X, m, \mathscr{F}, \mathscr{E})$ and $(\tilde{X}, \tilde{m}, \tilde{F}, \mathscr{E})$. The concepts corresponding to the latter will be denoted with tilde .

Definition 2.1. A mapping $q$ defined q.e. on $X$ taking values in $\tilde{X}$ is said to be a quasi-homeomorphism between $X$ and $\tilde{X}$ if, for any $\varepsilon>0$, there exist closed sets $F \subset X, \tilde{F} \subset \tilde{X}$ such that $\operatorname{Cap}(X-F)<\varepsilon, \operatorname{Cap}^{\sim}(\tilde{X}-\tilde{F})<\varepsilon$ and the restriction of $q$ to $F$ is a homeomorphism onto $\widetilde{F}$. $X$ and $\tilde{X}$ are said to be quasi-homeomorphic if there exists a quasi-homeomorphism between $X$ and $\tilde{X}$.

It is clear that $q$ is a quasi-homeomorphism if and only if there exist increasing sequences of closed sets $F_{k} \subset X$ and $\tilde{F}_{k} \subset \tilde{X}$ with $\lim _{k \rightarrow+\infty} \operatorname{Cap}\left(X-F_{k}\right)=0$, $\lim _{k \rightarrow+\infty} \operatorname{Cap}^{\sim}\left(\tilde{X}-\widetilde{F}_{k}\right)=0$ such that $q$ is one-to-one from $X_{0}=\bigcup_{k=1}^{\infty} F_{k}$ onto $\tilde{X}_{0}=\bigcup_{k=1}^{\infty} \widetilde{F}_{k}$ and its restriction to each $F_{k}$ is a homeomorphism onto $\widetilde{F}_{k}$. The domain of definition of a quasi-homeomorphism $q$ will always be considered to be such an $F_{\sigma}$-set $X_{0} . q$ and $q^{-1}$ are then Borel measurable transformations between $X_{0}$ and $\tilde{X}_{0}$. Hence the images by $q$ and $q^{-1}$ of analytic sets are also analytic sets $\left({ }^{8}\right)$.

${ }^{8}{ }^{8}$ Cf. [15, III, T11]. 
A quasi-homeomorphism $q$ is said to be capacity preserving if, for any analytic set $A \subset X_{0}$,

$$
\operatorname{Cap}(A)=\operatorname{Cap}^{\sim}(q(A))\left({ }^{9}\right) .
$$

We will write as $X \cong \tilde{X}$ if there exists a capacity preserving quasi-homeomorphism between $X$ and $\tilde{X}$.

LEMMA 2.1. Consider the underlying spaces $X, \hat{X}, \tilde{X}$ of three regular D-spaces. If $X \cong \hat{X}$ and $\hat{X} \cong \tilde{X}$, then $X \cong \tilde{X}$.

Proof. Suppose that $X$ and $\hat{X}$ (resp. $\hat{X}$ and $\hat{X}$ ) are related by the map $q_{1}$ (resp. $q_{2}$ ). For any $\varepsilon>0$, there exist closed sets $F \subset X, \hat{F}_{1} \subset \hat{X}, \hat{F}_{2} \subset \hat{X}$ and $\tilde{F} \subset \tilde{X}$ satisfying the following: $\operatorname{Cap}(X-F)<\varepsilon, \operatorname{Cap}^{\wedge}\left(\hat{X}-\hat{F}_{1}\right)<\varepsilon, \operatorname{Cap}^{\wedge}\left(\hat{X}-\hat{F}_{2}\right)<\varepsilon, \operatorname{Cap}^{\sim}(\tilde{X}-\tilde{F})<\varepsilon$ and $q_{1}$ (resp. $q_{2}$ ) is homeomorphic from $F$ (resp. $\hat{F}_{2}$ ) onto $\hat{F}_{1}$ (resp. $\tilde{F}$ ). Put $F^{\prime}$ $=q_{1}^{-1}\left(\hat{F}_{1} \cap \hat{F}_{2}\right)$ and $\tilde{F}^{\prime}=q_{2}\left(\hat{F}_{1} \cap \hat{F}_{2}\right)$. Then, $q=q_{2} \cdot q_{1}$ is homeomorphic from $F^{\prime}$ onto $\tilde{F}^{\prime}$ and

$$
\begin{aligned}
\operatorname{Cap}\left(X-F^{\prime}\right) & \leqq \operatorname{Cap}(X-F)+\operatorname{Cap}\left(q_{1}^{-1}\left(\hat{F}_{1}-\hat{F}_{2}\right)\right) \\
& =\operatorname{Cap}(X-F)+\operatorname{Cap}^{\wedge}\left(\hat{F}_{1}-\hat{F}_{2}\right)<2 \varepsilon .
\end{aligned}
$$

In the same way, we have $\operatorname{Cap}^{\sim}\left(\tilde{X}-\tilde{F}^{\prime}\right)<2 \varepsilon$. Thus, $X$ and $\tilde{X}$ are quasi-homeomorphic by the map $q$. Evidently $q$ is capacity preserving.

According to Definition 4.1 of [10], two $D$-spaces $(X, m, \mathscr{F}, \mathscr{E})$ and $(\tilde{X}, \tilde{m}, \mathscr{F}, \mathscr{E})$ are called equivalent if there exists an algebraic isomorphism $\Phi$ from $\mathscr{F} \cap L^{\infty}(X ; m)$ onto $\tilde{F} \cap L^{\infty}(\tilde{X} ; \tilde{m})$ which preserves three kinds of metrics- $L^{\infty}$-norm, $L^{2}$-norm and $\mathscr{E}$-norm. Notice that we always regard the normed algebra $\mathscr{F} \cap L^{\infty}(X ; m)$ (resp. $\tilde{F} \cap L^{\infty}(\tilde{X} ; \tilde{m})$ ) as the set of equivalence classes in the sense that two functions of $\mathscr{F} \cap L^{\infty}(X ; m)$ (resp. $\mathscr{F} \cap L^{\infty}(\tilde{X} ; \tilde{m})$ ) are identified if they coincide $m$-a.e. ( $\tilde{m}$-a.e.). The isomorphism $\Phi$ is viewed to transform each equivalence class to an equivalence class.

The isomorphism $\Phi$ can be uniquely extended to three kinds of transformations: a unitary map $\Phi_{1}$ from $\left(\mathscr{F}, \mathscr{E}^{\alpha}\right)$ onto $\left(\tilde{\mathscr{F}}, \mathscr{E}^{\alpha}\right)$, a unitary map $\Phi_{2}$ from $L_{0}^{2}(X)$ onto $L_{0}^{2}(\tilde{X})$ and an isometric isomorphism $\Phi_{3}$ from $L_{0}^{\infty}(X)$ onto $L_{0}^{\infty}(\tilde{X})$, where $L_{0}^{2}(X)$ (resp. $L_{0}^{\infty}(X)$ ) is the closure of $\mathscr{F} \cap L^{\infty}(X)$ in the metric space $L^{2}(X)$ (resp. $L^{\infty}(X)$ ). $L_{0}^{2}(\tilde{X})$ and $L_{0}^{\infty}(\tilde{X})$ are defined in the same way. Suppose that two $D$-spaces are regular. Then $\Phi_{1}$ is regarded as a unitary map from $\left(\mathscr{F}^{*}, \mathscr{E}^{\alpha}\right)$ onto $\left(\mathscr{F}^{*}, \mathscr{E}^{\alpha}\right)$, two functions being identified if they coincide q.e. Moreover we have in this case $L_{0}^{2}(X)=L^{2}(X)$ and $L_{0}^{\infty}(X) \supset C(X)$ because $\mathscr{F} \cap C(X)$ is dense in the metric space $L^{2}(X)$ (resp. $C(X)$ ) (see (5.4) of [10]). We also have $L_{0}^{2}(\tilde{X})=L^{2}(\tilde{X})$ and $L_{0}^{\infty}(\tilde{X}) \supset C(\tilde{X})$.

Now we will state the theorem of this section.

$\left.{ }^{(}\right)$This definition does not depend on the choice of set $X_{0}$. 
THEOREM 2.1. Assume that two regular D-spaces $(X, m, \mathscr{F}, \mathscr{E})$ and $(\tilde{X}, \tilde{m}, \mathscr{F}, \mathscr{E})$ are equivalent under an isomorphism $\Phi$. Then $X \cong \tilde{X}$ under a capacity preserving quasi-homeomorphism $q$ which has the following properties.

(q.1) $q$ induces the extension of the given isomorphism $\Phi:$ put

$$
\left(\Phi^{*} u\right)(x)=u\left(q^{-1} \tilde{x}\right),
$$

where $u$ is a function on $X$ and $\tilde{x}$ is a point of $\tilde{X}$ for which $u\left(q^{-1} \tilde{x}\right)$ makes sense, then $\Phi^{*}$ defines a transformation of functions which coincides on $\mathscr{F}^{*}$ with $\Phi_{1}$.

(q.2) $q$ is m-measure preserving: $m(A)=m(q(A))$ for any Borel set $A \subset X_{0}$.

Before proceeding to the proof of Theorem 2.1, we need several notions related to a regular $D$-space $(X, m, \mathscr{F}, \mathscr{E})$. For a set $A \subset X$, we put

$$
A^{\prime}=\{x \in X ; m(U(x) \cap A) \neq 0 \text { for every neighbourhood } U(x) \text { of } x\} .
$$

Obviously $A^{\prime}$ is closed. We say a closed set $F$ is $m$-regular if $F=F^{\prime}$.

Consider any closed set $F$. Then $F^{\prime}$ is a closed set contained in $F, m\left(F-F^{\prime}\right)=0$ and Cap $\left(X-F^{\prime}\right)=$ Cap $(X-F)$. We can see this in the same manner as in the proof of Theorem 1.2(ii). Furthermore $F^{\prime}$ is necessarily $m$-regular because $m\left(U(x) \cap F^{\prime}\right)$ $\geqq m(U(x) \cap F)-m\left(F-F^{\prime}\right)>0$ for any neighborhood $U(x)$ of $x \in F^{\prime}$.

Denote by $X^{\Delta}$ the compact space obtained from $X$ by adjoining the point at infinity $\Delta$. If $X$ is already compact, we regard $\Delta$ to be isolated. For each set $A \subset X$, we put $A^{\Delta}=A \cup \Delta$ and consider this to be a topological subspace of $X^{\Delta}$. A set $F \subset X$ is closed in $X$ if and only if $F^{\Delta}$ is compact.

We further use the notion $|u|_{A}$ defined by $|u|_{A}=\sup _{x \in A}|u(x)|$ for a function $u$ on $A \subset X$. Since $m$ is everywhere dense, we have

$$
\|u\|_{\infty}=|u|_{X}, \quad u \in C(X) .
$$

Finally let $\left\{F_{k}\right\}$ be an increasing sequence of $m$-regular closed sets of $X$ such that Cap $\left(X-F_{k}\right) \rightarrow 0$. Put $C\left(\left\{F_{k}\right\}\right)=\left\{u ; u\right.$ is defined on $X_{0}=\bigcup_{k=1}^{\infty} F_{k},|u|_{x_{0}}$ is finite, the restriction of $u$ to each $F_{k}$ is continuous there and continuously extendable to $F_{k}^{\Delta}$ by setting $\left.u(\Delta)=0\right\}$. Obviously $C(X) \subset C\left(\left\{F_{k}\right\}\right) \subset L^{\infty}(X ; m) . C\left(\left\{F_{k}\right\}\right)$ is a Banach algebra with norm ||$_{x_{0}}$. Further

$$
\|u\|_{\infty}=|u|_{x_{0}}, \quad u \in C\left(\left\{F_{k}\right\}\right) .
$$

This is clear from $m$-ess-sup $x_{x \in F_{k}}|u(x)|=|u|_{F_{k}}, k=1,2, \ldots$, which is due to the definition of $m$-regularity of $F_{k}$.

Each element $u$ of $C(X)$ (resp. $C\left(\left\{F_{k}\right\}\right)$ ) will always be regarded as a function on $X^{\Delta}$ (resp. $X_{0}^{\Delta}$ ) by setting $u(\Delta)=0$.

LEMMA 2.2. Let $Q$ be any countable subcollection of $\mathscr{F} \cap L^{\infty}$. Then there exists an increasing sequence of m-regular closed sets $F_{k}$ with $\mathrm{Cap}\left(X-F_{k}\right) \rightarrow 0$ such that each element of $Q$ has a unique modification belonging to $C\left(\left\{F_{k}\right\}\right)$. 
Proof. For $Q=\left\{u_{n}\right\}, u_{n} \in \mathscr{F} \cap L^{\infty}, n=1,2, \ldots$, we denote by $u_{n}^{*}$ a quasicontinuous modification of $u_{n}$ specified in Theorem 1.3. Thanks to the countable subadditivity of the capacity, we can select an increasing sequence of closed sets $F_{k} \subset X$ with Cap $\left(X-F_{k}\right) \rightarrow 0$ such that every function $u_{n}^{*}$ has the following property: the restriction of $u_{n}^{*}$ to each $F_{k}$ is continuous there. By virtue of the special manner of the construction of $u_{n}^{*}$ stated in Theorem 1.3, we may further assume that $u_{n}^{*}$ is continuously extendable from $F_{k}$ to $F_{k}^{\Delta}$ by setting $u_{n}^{*}(\Delta)=0$. In order to complete the proof of Lemma 2.2 , we only have to replace $F_{k}$ with its $m$-regularization $F_{k}^{\prime}$. After the replacement, we can see by (2.5) that $u_{n}^{*}$ becomes a unique element of $C\left(\left\{F_{k}\right\}\right)$ which coincides with $u_{n} m$-a.e.

Now we will prove Theorem 2.1 by means of the next three lemmas.

LEMMA 2.3. Under the assumption of Theorem 2.1, there exists an increasing sequence of m-regular closed sets $F_{k} \subset X, k=1,2, \ldots$, with $\lim _{k \rightarrow+\infty} \operatorname{Cap}\left(X-F_{k}\right)=0$ which satisfies the following. We put $X_{0}=\bigcup_{k=1}^{\infty} F_{k}$.

(i) There is an algebraic isomorphic and isometric transformation $\psi$ from $\left(C(\tilde{X}),||_{\tilde{X}}\right)$ into $\left(C\left(\left\{F_{k}\right\}\right),||_{x_{0}}\right) . \psi$ is just the restriction of the transform $\Phi_{3}^{-1}$ to $C(\tilde{X})$.

(ii) There is a mapping $q$ from $X_{0}^{\Delta}$ into $\tilde{X}^{\tilde{\Delta}}$ such that $q(\Delta)=\tilde{\Delta}$ and the restriction of $q$ to each $F_{k}^{\Delta}$ is continuous there. For each $x \in X^{\Delta}, q x$ is characterized by

$$
\tilde{u}(q x)=(\psi \tilde{u})(x), \quad \tilde{u} \in C(\tilde{X}) .
$$

Proof. (i) Since $\tilde{F} \cap C(\tilde{X})$ is a dense subalgebra of $C(\tilde{X})$, we can find a countable subset $\tilde{C}_{1} \subset \mathscr{F} \cap C(\tilde{X})$ such that the algebra $\mathscr{A}\left(\tilde{C}_{1}\right)$ generated by $\tilde{C}_{1}$ is dense in $C(\tilde{X})$ with maximum norm. Applying Lemma 2.2 to $\Phi^{-1} \widetilde{C}_{1} \subset \mathscr{F} \cap L^{\infty}(X ; m)$, we get an increasing sequence $\left\{F_{k}\right\}$ of $m$-regular closed sets of $X$ with Cap $\left(X-F_{k}\right)$ $\rightarrow 0$ such that, for every $\tilde{u} \in \widetilde{C}_{1}, \Phi^{-1} \tilde{u}$ has a unique modification belonging to $C\left(\left\{F_{k}\right\}\right)$. Denote this modification by $\psi \tilde{u}$. The map $\psi$ is extended to an algebraic isomorphism on $\mathscr{A}\left(\widetilde{C}_{1}\right)$ which is consistent because of

$$
|\tilde{u}|_{\tilde{x}}=|\psi \tilde{u}|_{x_{0}}, \quad \tilde{u} \in \mathscr{A}\left(\tilde{C}_{1}\right),
$$

where $X_{0}=\bigcup_{k=1}^{\infty} F_{k}$. The equality (2.7) follows from (2.4) and (2.5) as $|\tilde{u}|_{X}$ $=\|\tilde{u}\|_{\infty}=\left\|\Phi_{3}^{-1} \tilde{u}\right\|_{\infty}=|\psi \tilde{u}|_{X_{0}}$. Now $\psi$ is readily extended to a map from $C(\tilde{X})$ into $C\left(\left\{F_{k}\right\}\right)$ satisfying conditions of the first statement of the present lemma.

(ii) For each $x \in X_{0}^{\Delta}, l_{x}(\tilde{u})=(\psi \tilde{u})(x), \tilde{u} \in C(\tilde{X})$, is a character (a linear multiplicative functional) on $C(\tilde{X})$. Hence there exists a unique element $q x \in \tilde{X}^{\tilde{\Delta}}$ such as $l_{x}(\tilde{u})=\tilde{u}(q x), \tilde{u} \in C(\tilde{X})$. Since $l_{\Delta}(\tilde{u}) \equiv 0$, we have $q \Delta=\tilde{\Delta}$. Suppose that $x_{n} \in F_{k}^{\Delta}$ converges to $x \in F_{k}^{\Delta}$. Then $\tilde{u}\left(q x_{n}\right)=(\psi \tilde{u})\left(x_{n}\right)$ converges to $(\psi \tilde{u})(x)=\tilde{u}(q x), \tilde{u} \in C(\tilde{X})$, which implies $q x_{n} \rightarrow q x, n \rightarrow \infty$, and hence the restriction of $q$ to $F_{k}^{\Delta}$ is continuous there.

LEMMA 2.4. In addition to the assumption of Theorem 2.1, we assume

$$
\Phi(\mathscr{F} \cap C(X)) \subset \mathscr{F} \cap C(\tilde{X}) .
$$


Then all the conclusions of Theorem 2.1 are valid for the map $q$ of Lemma 2.3.

Proof. By assumption (2.8), there exists an algebraic isomorphic and isometric transformation $\varphi$ from $C(X)$ into $C(\tilde{X}): \varphi$ is just the restriction of the transform $\Phi_{3}$ to $C(X) \subset L_{0}^{\infty}(X)$. Therefore there is a continuous map $\gamma$ from $\tilde{X}^{\tilde{\Delta}}$ onto $X^{\Delta}$ such that, for each $\tilde{x} \in \tilde{X}^{\tilde{\Delta}}, \gamma \tilde{X}$ is characterized by

$$
u(\gamma \tilde{x})=\varphi u(\tilde{x}), \quad u \in C(X) .
$$

On the other hand, the map $\psi$ of Lemma 2.3 is the inverse of $\varphi$ in the sense that $\psi \varphi u(x)=u(x), x \in X_{0}^{\Delta}$, for every $u \in C(X)$. Indeed $u \in C(X)$ and $\psi \varphi u\left(=\Phi_{3}^{-1} \cdot \Phi_{3} u\right)$ $\in C\left(\left\{F_{k}\right\}\right)$ are in the same class of $L_{0}^{\infty}(X)$ and so they are identical on $X_{0}^{\Delta}$ by virtue of (2.5). Hence, in view of (2.6) and (2.9), the map $\gamma$ is the inverse of $q$ of Lemma 2.3:

$$
\gamma \cdot q x=x, \quad x \in X_{0}^{\Delta} .
$$

In particular $q\left(X_{0}\right) \subset \tilde{X}$ because $\gamma(\tilde{\Delta})=\Delta$. We put

$$
\tilde{F}_{k}=q\left(F_{k}\right), \quad k=1,2, \ldots, \quad \tilde{X}_{0}=\bigcup_{k=1}^{\infty} \tilde{F}_{k} .
$$

Since the restriction of $q$ to the compactum $F_{k}^{\Delta \subset X^{\Delta}}$ is a continuous map, its image $q\left(F_{k}^{\Delta}\right)=\tilde{F}_{k}^{\tilde{\Delta}}$ is a compact set of $\tilde{X}^{\tilde{\Delta}} . \widetilde{F}_{k}$ is therefore a closed subset of $\tilde{X}$.

From now on, let us restrict the domain of the definition of $q$ (resp. $\gamma$ ) to $X_{0}$ (resp. $\tilde{X}$ ) and study the detailed properties they possess.

First of all we know from (2.10) that $q$ is one-to-one from $X_{0}$ onto $\tilde{X}_{0}$ and its restriction to each $F_{k}$ is a homeomorphism onto $\tilde{F}_{k}$, the inverse being $\gamma$.

We will prove that $q$ is measure preserving between $X_{0}$ and $\tilde{X}_{0}$. It is enough to show

$$
m\left(q^{-1}(\tilde{K})\right)=\tilde{m}(\tilde{K})
$$

for any compact set $\tilde{K}$ contained in some $\widetilde{F}_{k}$. To see $(2.12)$, choose a sequence $\tilde{u}_{n} \in \tilde{F} \cap C(\tilde{X})$ converging to the indicator function of $\widetilde{K}$ everywhere on $\tilde{X}$ as well as in $L^{2}(\tilde{X} ; \tilde{m})$-sense. This is possible because $\tilde{F} \cap C(\tilde{X})$ is a lattice and a dense subset i f $C(\tilde{X})$. Then $\psi \tilde{u}_{n}(x)=\tilde{u}_{n}(q x)$ converges to the indicator function of $q^{-1}(\tilde{K})$ $\subset F_{k}$ for each $x \in X_{0}$ and hence $m$-a.e. on $X$. Since $\psi$ on $\tilde{F} \cap C(\tilde{X})$ is a modification of $\Phi^{-1}$ which preserves $L^{2}$-norm, $\left\{\psi \tilde{u}_{n}\right\}$ also forms a Cauchy sequence in $L^{2}(X ; m)$ and further

$$
\tilde{m}(\tilde{K})=\lim _{n \rightarrow+\infty}\left(\tilde{u}_{n}, \tilde{u}_{n}\right)_{\tilde{X}}=\lim _{n \rightarrow+\infty}\left(\psi \tilde{u}_{n}, \psi \tilde{u}_{n}\right)_{X}=m\left(q^{-1}(\tilde{K})\right),
$$

getting (2.12).

Exactly in the same way as above, we can prove

$$
m(K)=\tilde{m}\left(\gamma^{-1}(K)\right)
$$


for any compact set $K \subset X$. Moreover, combining (2.12) and (2.13), we come to the conclusion that

$$
\tilde{m}\left(\gamma^{-1}\left(F_{k}\right)-\tilde{F}_{k}\right)=0, \quad k=1,2, \ldots
$$

Indeed, fix a number $k$ and take any compact set $\tilde{K} \subset \gamma^{-1}\left(F_{k}\right)-\widetilde{F}_{k}$. Then put $K=\gamma(\tilde{K})$ and $\tilde{K}_{1}=q(K) . K$ and $\tilde{K}_{1}$ are compact sets in $F_{k}$ and $\tilde{F}_{k}$ respectively. Since $\gamma^{-1}(K) \supset \widetilde{K} \cup \widetilde{K}_{1}$, we have

$$
\tilde{m}\left(\tilde{K}_{1}\right)=m\left(q^{-1}\left(\tilde{K}_{1}\right)\right)=m(K)=\tilde{m}\left(\gamma^{-1}(K)\right) \geqq \tilde{m}\left(\tilde{K} \cup \tilde{K}_{1}\right)
$$

from which follows $\tilde{m}(\tilde{K})=0$.

Next we have to show

$$
\tilde{m}\left(\gamma^{-1}(\Delta)\right)=0 .
$$

Observe that $\gamma^{-1}(\Delta)=\{\tilde{x} \in \tilde{X} ; \varphi u(\tilde{x})=0$ for every $u \in C(X)\}$. Notice further that, since $\mathscr{F} \cap C(X)$ is dense in $L^{2}(X ; m)$, the space $\varphi(\mathscr{F} \cap C(X))(=\Phi(\mathscr{F} \cap C(X)))$ is dense in $L^{2}(\tilde{X} ; \tilde{m})\left(=\Phi_{2}\left(L^{2}(X ; m)\right)\right)$. Hence for any compactum $\tilde{K} \subset \gamma^{-1}(\Delta)$ there is a sequence $u_{n} \in \mathscr{F} \cap C(X)$ such that $\varphi u_{n}$ converges $\tilde{m}$-a.e. on $\tilde{X}$ to the indicator function of $\tilde{K}$. But $\varphi u_{n}(\tilde{x})=0, \tilde{x} \in \tilde{K}, n=1,2, \ldots$, and we have $\tilde{m}(\tilde{K})=0$ proving (2.15).

We are in a position to complete the proof of Lemma 2.4. Let us derive the inequality

$$
\operatorname{Cap}^{\sim}(\tilde{K}) \leqq \operatorname{Cap}(K)
$$

where $\tilde{K}$ is any compact subset of $\gamma^{-1}(X)$ and $K=\gamma(\tilde{K})$. Since $\gamma$ is continuous, $K$ is a compact set of $X$. Consider the sets $\mathscr{C}_{K}=\{u \in \mathscr{F} \cap C(X) ; u \geqq 1$ on $K\}$ and $\widetilde{\mathscr{C}}_{\tilde{K}}=\{\tilde{u} \in \tilde{F} \cap C(\tilde{X}) ; \tilde{u} \geqq 1$ on $\tilde{K}\}$, and observe the inclusion $\varphi\left(\mathscr{C}_{K}\right) \subset \mathscr{C}_{\tilde{K}}$. Since $\varphi$

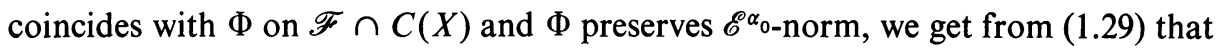

$$
\begin{aligned}
\operatorname{Cap}(K) & =\inf _{u \in \mathscr{C}_{K}} \mathscr{E} \alpha_{0}(u, u)=\inf _{u \in \mathscr{C}_{K}} \mathscr{E}^{\alpha_{\alpha}}(\varphi u, \varphi u) \\
& =\inf _{\left.\tilde{u} \in \mathscr{C}_{K}\right)} \mathscr{E}_{\boldsymbol{E}_{0}}(\tilde{u}, \tilde{u}) \geqq \operatorname{Cap}^{\sim}(\tilde{K}) .
\end{aligned}
$$

We can now show that $q$ is capacity preserving on $X_{0}$. On account of Theorem 1.1(b) and (1.5), it suffices to prove for any compact subset $K \subset F_{k}$ with a fixed $k$,

$$
\operatorname{Cap}(K)=\operatorname{Cap}^{\sim}(\tilde{K})
$$

where $\widetilde{K}=q(K)$. Noting the inclusion

$$
\psi\left(\widetilde{\mathscr{C}}_{\tilde{K}}\right) \subset\left\{u \in \mathscr{F} \cap C\left(\left\{F_{k}\right\}\right) ; u \geqq 1 \text { on } K\right\} \subset \mathscr{L}_{K}^{*},
$$

we have

$$
\begin{aligned}
\operatorname{Cap}^{\sim}(\tilde{K}) & =\inf _{\tilde{u} \in \tilde{\mathscr{E}}_{K}} \mathscr{E}^{\mathscr{E}_{0}} 0(\tilde{u}, \tilde{u})=\inf _{\tilde{u} \in \tilde{\mathscr{E}} \tilde{K}} \mathscr{E}^{\alpha_{0}}(\psi \tilde{u}, \psi \tilde{u}) \\
& =\inf _{u \in \psi(\tilde{\mathscr{E}} \tilde{K})} \mathscr{E} \alpha_{0}(u, u) \geqq \operatorname{Cap}(K),
\end{aligned}
$$

which combined with (2.16), proves (2.17). 
For the proof that $q$ is a capacity preserving quasi-homeomorphism and measure preserving, it only remains to show

$$
\operatorname{Cap}^{\sim}\left(\tilde{X}-\tilde{X}_{0}\right)=0 \text {. }
$$

Choose any $\varepsilon>0$ and fix a number $k$ such as Cap $\left(X-F_{k}\right)<\varepsilon$. We are going to show

$$
\operatorname{Cap}^{\sim}\left(\tilde{X}-\widetilde{F}_{k}\right)<\varepsilon .
$$

Observe that $\tilde{X}-\tilde{F}_{k}$ is an open set of $\tilde{X}$ consisting of three disjoint parts: $\tilde{X}-\widetilde{F}_{k}$ $=\gamma^{-1}\left(X-F_{k}\right)+\left(\gamma^{-1}\left(F_{k}\right)-\tilde{F}_{k}\right)+\gamma^{-1}(\Delta)$. By (2.14) and (2.15), $\tilde{m}$-measures of the last two terms of the right-hand side are zero. $\gamma^{-1}\left(X-F_{k}\right)$ is open and contained in $\tilde{X}-\tilde{F}_{k}$. Hence by definition $(0.1)$ of the capacity, we have

$$
\operatorname{Cap}^{\sim}\left(\tilde{X}-\tilde{F}_{k}\right)=\operatorname{Cap}^{\sim}\left(\gamma^{-1}\left(X-F_{k}\right)\right) .
$$

On the other hand (2.16) and (1.5) mean the following:

$$
\begin{aligned}
\operatorname{Cap}^{\sim}\left(\gamma^{-1}\left(X-F_{k}\right)\right) & =\sup _{\tilde{K}} \operatorname{Cap}^{\sim}(\tilde{K}) \leqq \sup _{K=\gamma(\tilde{K})} \operatorname{Cap}(K) \\
& \leqq \operatorname{Cap}\left(X-F_{k}\right)<\varepsilon,
\end{aligned}
$$

the supremum being taken for all compact set $\tilde{K} \subset \gamma^{-1}\left(X-F_{k}\right)$. Thus we arrive at (2.19).

It is easy to see that our $q$ possesses the property (q.1) of Theorem $2.1:(2.9)$ and (2.10) mean, for $u \in \mathscr{F} \cap C(X)$,

$$
\Phi^{*} u=\Phi_{1} u \text { q.e., }
$$

which can be extended to $\mathscr{F}^{*}$ by virtue of Lemma 1.2 . We have completed the proof of Lemma 2.4 .

LEMMA 2.5. Under the assumption of Theorem 2.1, there exists a regular D-space $(\hat{X}, \hat{m}, \hat{F}, \hat{\mathscr{E}})$ satisfying the following:

(1) Both the given regular D-spaces are equivalent to $(\hat{X}, \hat{m}, \hat{\mathscr{F}}, \hat{\mathscr{E}})$ by isomorphisms, say, $\Phi^{\prime}$ and $\Phi^{\prime \prime} . \Phi$ is equal to $\left(\Phi^{\prime \prime}\right)^{-1} \cdot \Phi^{\prime}$.

(2) $\Phi^{\prime}(\mathscr{F} \cap C(X)) \subset \hat{\mathscr{F}} \cap C(\hat{X}), \Phi^{\prime \prime}(\tilde{\mathscr{F}} \cap C(\tilde{X})) \subset \hat{\mathscr{F}} \cap C(\hat{X})$.

Proof. This lemma is an application of the regular representation theorem of [10]. First of all we will establish the inclusion

$$
\Phi_{3}\left(C_{0}(X)\right) \subset L^{1}(\tilde{X} ; \tilde{m}), \quad \Phi_{3}^{-1}\left(C_{0}(\tilde{X})\right) \subset L^{1}(X ; m) .
$$

It is enough to prove the first. For any function $u \in C_{0}(X)$, there is a nonnegative function $v \in \mathscr{F} \cap C(X)$ such as $v \geqq \sqrt{ }|u|$ on $X$. Since $\Phi_{3}$ is a lattice isomorph as well as an algebraic isomorph and since $\Phi_{3} v \in \tilde{F} \subset L^{2}(\tilde{X} ; \tilde{m})$, we have $\Phi_{3}(\sqrt{ }|u|)$ $\in L^{2}(\tilde{X} ; \tilde{m})$ and $\left|\Phi_{3} u\right|=\left(\Phi_{3}(\sqrt{ }|u|)\right)^{2} \in L^{1}(\tilde{X} ; \tilde{m})$.

Now denote by $L$ the closed subalgebra in $L_{0}^{\infty}(X)$ generated by $C(X) \cup \Phi_{3}^{-1} C(\tilde{X})$. Then $L$ satisfies the condition (C) of $[10, \S 5]$. (C.1) and (C.2) are clear. By (2.22), $L^{1}(X ; m) \cap L$ includes the algebra generated by $C_{0}(X) \cup \Phi_{3}^{-1}\left(C_{0}(\tilde{X})\right)$ which is 
dense in $L$, proving (C.3). Therefore we can take as $(\hat{X}, \hat{m}, \hat{\mathscr{F}}, \hat{\mathscr{E}})$ the regular representation of $(X, m, \mathscr{F}, \mathscr{E})$ with respect to $L$ (Theorem 2 of [10]). The algebraic isomorphism $\Phi^{\prime}$ associated with this representation is translating $\mathscr{F} \cap L$ onto $\hat{\mathscr{F}} \cap C(\hat{X})$ getting the first inclusion of (2). The second is also clear because $(\hat{X}, \hat{m}, \hat{\mathscr{F}}, \hat{\mathscr{E}})$ is the regular representation of $(\tilde{X}, \tilde{m}, \tilde{\mathscr{F}}, \mathscr{E})$ with respect to $\tilde{L}$ under the isomorphism $\Phi^{\prime} \cdot \Phi^{-1}, \tilde{L}$ being the closed subalgebra of $L_{0}^{\infty}(\tilde{X} ; \tilde{m})$ generated by $\Phi(C(X)) \cup C(\tilde{X})$.

Proof of Theorem 2.1. Lemmas 2.1, 2.4 and 2.5 admit us to conclude that $X \cong \tilde{X}$ under a capacity preserving quasi-homeomorphism $q$ possessing the property (q.1). (q.2) is a consequence of (q.1) because $\Phi^{*}$ is $L^{2}$-norm preserving from $\mathscr{F} *$ onto $\tilde{\mathscr{F}}^{*}$. The proof of Theorem 2.1 is complete.

If two $D$-spaces are equivalent and if one of them is regular, then it is said to be a regular representation of the other.

COROLlary to TheOREM 2.1. The underlying space of a regular representation of a given D-space is unique up to a capacity preserving quasi-homeomorphism.

3. Potential theory for symmetric Ray processes. Let $(X, m, \mathscr{F}, \mathscr{E})$ be a strongly regular $D$-space and $\left\{R_{\alpha}(x, E), \alpha>0\right\}$ be its associated symmetric Ray resolvent kernel on $X$. For a function $u$ on $X$, put

$$
R_{\alpha} u(x)=\int_{X} R_{\alpha}(x, d y) u(y), \quad x \in X,
$$

whenever the right-hand side makes sense. The images by $R_{\alpha}$ of Borel (universally) measurable functions are also Borel (universally) measurable. By definition, $(\mathscr{F}, \mathscr{E})$ is generated by $\left\{R_{\alpha}(x, E), \alpha>0\right\}$, that is, $R_{\alpha}\left(L^{2}(X ; m) \cap C(X)\right) \subset \mathscr{F} \cap C(X)$ and $R_{\alpha} u, u \in L^{2}(X ; m) \cap C(X)$, satisfies the equation

$$
\mathscr{E}^{\alpha}\left(R_{\alpha} u, v\right)=(u, v)_{X}
$$

for every $v \in \mathscr{F}$. Moreover $\mathscr{F} \cap C(X)$ includes a set $C_{1}$ attached to the Ray resolvent (Definition 2.5 of [10]).

3.1. Supermedian and excessive functions.

LEMMA 3.1. For any nonnegative measurable function $u$ of $L^{2}(X ; m)$, the function $R_{\alpha} u$ defined by (3.1) belongs to the space $\mathscr{F}^{*}$ and satisfies the equation (3.2) for each $\alpha>0$.

We will prove this lemma by making use of the following proposition:

Proposition. Suppose that a set $H$ of real-valued functions on $X$ satisfies the next conditions.

(H.1) If $f_{1}, f_{2} \in H$ and $c_{1} f_{1}+c_{2} f_{2} \geqq 0$ with some constants $c_{1}, c_{2}$, then $c_{1} f_{1}$ $+c_{2} f_{2} \in H$.

(H.2) If $f_{n} \in H$ increases to $f \in L^{2}(X ; m)$, then $f \in H$.

(H.3) $C_{0}^{+}(X) \subset H$.

Then $H$ contains all nonnegative Borel measurable functions of $L^{2}(X ; m)$. 
For the proof of the Proposition, it is enough to take any open set $E \subset X$ with compact closure and consider the class $S$ of all Borel subsets of $E$ whose indicator functions are in $H$. $S$ contains all open subsets of $E$. Since $S$ is a $\lambda$-system relative to $E$, it contains all Borel subsets of $E$ (Lemma 0.1 of [7]). The rest of the proof is clear.

Proof of Lemma 3.1. Let $H$ be the set of all nonnegative Borel measurable functions $u$ of $L^{2}(X ; m)$ such that $R_{\alpha} u$ belongs to $\mathscr{F}^{*}$ and satisfies equation (3.2) for each $\alpha>0$. $H$ satisfies (H.3) because $L^{2}(X ; m) \cap C(X) \subset H$. Suppose that $u_{n} \in H$ increases to $u \in L^{2}(X ; m)$. Then

$$
\begin{aligned}
\mathscr{E}^{\alpha}\left(R_{\alpha} u_{n}-R_{\alpha} u_{m}, R_{\alpha} u_{n}-R_{\alpha} u_{m}\right) & =\left(u_{n}-u_{m}, R_{\alpha}\left(u_{n}-u_{m}\right)\right)_{X} \\
& \leqq(1 / \alpha)\left(u_{n}-u_{m}, u_{n}-u_{m}\right)_{X} \rightarrow 0, \quad n, m \rightarrow+\infty .
\end{aligned}
$$

By virtue of Lemma 1.2, a subsequence of $R_{\alpha} u_{n} \in \mathscr{F}^{*}$ converges to a function $\mathscr{F} *$ q.e. on $X$ as well as in $\mathscr{E}^{\alpha}$-norm. However $R_{\alpha} u_{n}(x)$ converges to $R_{\alpha} u(x)$ for each $x \in X$. Therefore $R_{\alpha} u$ belongs to $\mathscr{F}^{*}$ and satisfies (3.2). Condition (H.2) is verified. Thus we see by the proposition that Lemma 3.1 is valid for any nonnegative Borel measurable function $u$ of $L^{2}(X ; m)$.

Finally let $u$ be a nonnegative universally measurable function of $L^{2}(X ; m)$. There exist nonnegative Borel measurable functions $u_{1}$ and $u_{2}$ such that $u_{1} \leqq u \leqq u_{2}$ on $X$ and $u_{1}=u_{2} m$-a.e. on $X$. We have $R_{\alpha} u_{1} \leqq R_{\alpha} u \leqq R_{\alpha} u_{2}$ on $X$. Further, by the symmetry of $R_{\alpha}$,

$$
\begin{aligned}
0 & \leqq \int_{X}\left(R_{\alpha} u_{2}-R_{\alpha} u_{1}\right)(x) m(d x)=\int_{X} R_{\alpha} 1(x)\left(u_{2}(x)-u_{1}(x)\right) m(d x) \\
& \leqq \frac{1}{\alpha} \int_{X}\left(u_{2}(x)-u_{1}(x)\right) m(d x)=0
\end{aligned}
$$

which implies $R_{\alpha} u_{1}=R_{\alpha} u_{2} m$-a.e. on $X$. Since $R_{\alpha} u_{1}$ and $R_{\alpha} u_{2}$ are quasi-continuous, we see by Theorem 1.2 (ii) that $R_{\alpha} u_{1}=R_{\alpha} u=R_{\alpha} u_{2}$ q.e. on $X$ and consequently $R_{\alpha} u \in \mathscr{F}^{*}$. The equation (3.2) for $u$ can be derived from that for $u_{1}$. The proof of Lemma 3.1 is complete.

Definition 3.1. A function $u$ on $X$ is said to be $\left(\alpha_{0^{-}}\right)$supermedian if the following two conditions are satisfied:

(3.3) $u$ is nonnegative and universally measurable,

(3.4) $\beta R_{\beta+\alpha_{0}} u(x) \leqq u(x), x \in X, \beta>0$.

A supermedian function $u$ is said to be $\left(\alpha_{0^{-}}\right)$excessive if

(3.5) $\lim _{\beta \rightarrow+\infty} \beta R_{\beta+\alpha_{0}} u(x)=u(x), x \in X$.

If $u$ is nonnegative and universally measurable, then $R_{\alpha_{0}} u$ is excessive.

If $u$ is a nonnegative universally measurable function and $\lim _{\beta \rightarrow \infty} \beta R_{\beta+\alpha_{0}} u(x)$ $=\tilde{u}(x)$ exists for every $x \in X$, then the limit function $\tilde{u}$ is said to be the regularization of $u$. Every supermedian function has its regularization which turns out to be excessive. 
THEOREM 3.1. If a function $u$ is nonnegative universally measurable, belongs to the space $\mathscr{F}$ and has its regularization $\tilde{u}$, then $\tilde{u}$ is a quasi-continuous modification of $u$. In particular any excessive function belonging to $\mathscr{F}$ is an element of $\mathscr{F} *$.

Proof. We see by Lemma 3.1, that $R_{\alpha} u \in \mathscr{F}^{*}$ and the operator $R_{\alpha}$ applied to $u$ is identical with $L^{2}$-resolvent associated with $(\mathscr{F}, \mathscr{E})$. Hence by taking Lemma 2.1(iii) of [10] and Lemma 1.2 of the present paper into account, we see that a subsequence of $\beta R_{\beta+\alpha_{0}} u$ converges q.e. on $X$ to a quasi-continuous modification of $u$. Thus we get Theorem 3.1.

REMARK 3.1. Every supermedian function belonging to the space $\mathscr{F} *$ is quasisupermedian in the sense of subsection 1.3. According to Theorem 3.1, every excessive function belonging to the space $\mathscr{F}$ is quasi-supermedian.

3.2. The associated Ray process and the branch set. Put $\bar{X}=X \cup \partial$ where $\partial$ is adjoined to $X$ as the point at infinity if $X$ is noncompact and as an isolated point if $X$ is compact. Extend the kernel $\left\{R_{\alpha}(x, E), \alpha>0\right\}$ to $\bar{X}$ in the manner of Remark 2.2(ii) of [10]. Then the extended kernel becomes a conservative Ray resolvent over the compactum $\bar{X}$ to which the original set $C_{1}$ is still attached if we extend each function $u$ of $C_{1}$ to $\bar{X}$ by setting $u(\partial)=0$.

Therefore the results of D. Ray [18, Theorem I, II and III] concerning resolvents on compact spaces and their improvements by H. Kunita and T. Watanabe [13, §2] can be brought over to our situation and we get the following conclusions.

The first conclusion is about the branch set. For each $x \in X$, the measure $\alpha R_{\alpha}(x, \cdot)$ on $X$ converges to a unique substochastic measure $\mu(x, \cdot)$ :

$$
\lim _{\alpha \rightarrow+\infty} \alpha R_{\alpha} f(x)=\int_{X} \mu(x, d y) f(y) \text { for any } f \in C(X) .
$$

A point $x \in X$ is said to be a branch point if the measure $\mu(x, \cdot)$ is not a unit distribution at $x$. The set $X_{b}$ of all branch points of $X$ is called the branch set. The measure $\mu(x, \cdot)$ is not supported by $X_{b}$ for any $x \in X . X_{b}$ is characterized as follows:

$$
X_{b}=\bigcup_{g \in C_{1}^{\prime}}\left\{x ; g(x)>\int_{X} g(y) \mu(x, d y)\right\},
$$

where $C_{1}^{\prime}=\left\{g=f \wedge c ; f \in C_{1}, c\right.$ is any positive rational number $\}$.

The second is about the transition function. There is a unique sub-Markov transition function $P_{t}(x, E)$ on $X$ such that

$$
P_{t} f(x)=\int_{X} P_{t}(x, d y) f(y), \quad f \in C(X), x \in X,
$$

defines a right continuous function of $t>0$ with

$$
\int_{0}^{+\infty} e^{-\alpha t} P_{t} f(x) d t=R_{\alpha} f(x), \quad \alpha>0 .
$$


The third is the existence of a right continuous strong Markov process on $X$ with transition function $P_{t}$. This is called the Ray process associated with $\left\{R_{\alpha}(x, E), \alpha>0\right\}$. We can adopt as the Ray process the canonical realization $\boldsymbol{M}=\left(W, \mathscr{B}_{t}^{0}, P_{x}\right)$ of $\left\{R_{\alpha}(x, E), \alpha>0\right\}$ in the following sense $\left({ }^{10}\right) . W$ consists of paths $\omega=\omega(t), t \in[0,+\infty)$, taking values in $\bar{X}$ such that $\omega(t)$ is right continuous in $t \in[0,+\infty)$, has the left limit at any $t \in(0,+\infty)$ and stays at $\partial$ after its lifetime $\zeta(\omega)$. The $t$ th coordinate $\omega(t)$ of $\omega$ is denoted by $X_{t}(\omega) . \zeta(\omega)$ is defined by $\inf \left\{t \geqq 0, X_{t}(\omega)=\partial\right\} . \mathscr{B}_{t}^{0}$ is the $\sigma$-field of subsets of $W$ generated by $\left\{X_{s} \in E\right\}$ with $0 \leqq s \leqq t$ and Borel set $E \subset \bar{X}$. For each $x, P_{x}$ is a unique probability measure on $\mathscr{B}^{0}=\bigvee_{t \geqq 0} \mathscr{B}_{t}^{0}$ which satisfies

$$
\begin{aligned}
P_{x}\left(X_{t_{1}}\right. & \left.\in E_{1}, X_{t_{2}} \in E_{2}, \ldots, X_{t_{n}} \in E_{n}\right) \\
& =\int_{E_{1}} \int_{E_{2}} \cdots \int_{E_{n}} \bar{P}_{t_{1}}\left(x, d y_{1}\right) \bar{P}_{t_{2}-t_{1}}\left(y_{1}, d y_{2}\right) \cdots \bar{P}_{t_{n}-t_{n-1}}\left(y_{n-1}, d y_{n}\right)
\end{aligned}
$$

for $0<t_{1}<t_{2}<\cdots<t_{n}$ and Borel sets $E_{1}, E_{2}, \ldots, E_{n}$ of $\bar{X}$, where $\bar{P}_{t}(x, E)$ $=P_{t}(x, E \cap X)+\left(1-P_{t}(x, X)\right) \delta_{\{\partial\}}(E)$.

The Ray process $\boldsymbol{M}=\left(W, \mathscr{B}_{t}^{0}, P_{x}\right)$ has the following properties:

(M.1) $P_{x}\left(X_{0} \in E\right)=\mu(x, E)$ for $x \in X$ and Borel $E \subset X$. Define $\mathscr{B}$ to be the completion of $\mathscr{B}^{0}$ with respect to the family of measures $\left\{P \mu(\cdot)=\int_{X} \mu(d x) P_{x}(\cdot) ; \mu\right.$ is a finite measure on $\bar{X}\}$ and $\mathscr{B}_{t}$ to be the completion of $\mathscr{B}_{t}^{0}$ in $\mathscr{B}$ with respect to the same family $\left({ }^{11}\right)$.

(M.2) Strong Markov property with respect to the augmented fields $\left\{\mathscr{B}_{t}\right\}$ : for any stopping time $T, t>0$ and Borel $E \subset \bar{X}, P_{x}\left(X_{T+t} \in E \mid \mathscr{B}_{T}\right)=P_{t}\left(X_{T}, E\right), P_{x^{-}}$ almost everywhere for each $x \in X$. Here, $T$ is said to be a stopping time if $\{T \leqq t\}$ $\in \mathscr{B}_{t}$ for any $t \geqq 0$ and $\mathscr{B}_{T}$ is defined as the collection of those sets $\Lambda \in \mathscr{B}$ such that $\{T \leqq t\} \cap \Lambda \in \mathscr{B}_{t}$ for all $t$.

(M.3) Quasi-left continuity in the restricted. sense: if stopping times $T_{n}$ increase to $T$, then $X_{T}=\lim _{n \rightarrow+\infty} X_{T_{n}} P_{x}$-almost everywhere on the set

$$
\left\{T<+\infty, \lim _{n \rightarrow+\infty} X_{T_{n}} \in \bar{X}-X_{b}\right\} \text { for each } x \in X .
$$

(M.4) $P_{x}\left(X_{t} \in \bar{X}-X_{b}\right.$ for any $\left.t \geqq 0\right)=1, x \in X$.

For a set $A \subset X$, we define the first entry time $\sigma_{A}(\omega)$ and the hitting time $\sigma_{A}^{\prime}(\omega)$ by

$$
\begin{aligned}
& \sigma_{A}(\omega)=\inf \left\{t \geqq 0 ; X_{t} \in A\right\}, \\
& \sigma_{A}^{\prime}(\omega)=\inf \left\{t>0 ; X_{t} \in A\right\} .
\end{aligned}
$$

We define $\sigma_{A}(\omega)$ or $\sigma_{A}^{\prime}(\omega)$ to be $\zeta(\omega)$ when the set in the braces is empty. If $A$ is analytic, then random times $\sigma_{A}, \sigma_{A}^{\prime}$ and $\tau=\inf \left\{t>0 ; X_{t-} \in A\right\}$ are $\mathscr{B}_{t}$-stopping times. We can see this by [15, Chapter IV, T47 and 53].

\footnotetext{
${ }^{(10)}$ See $[16, X I I I]$ for the canonical realization of a Feller semigroup.

(11) See R. M. Blumenthal and R. K. Getoor [2, p. 26] for the terminology.
} 
THEOREM 3.2. The branch set $X_{b}$ is polar in the sense of $\S 1$.

Proof. By Lemma 4.1 of [10] and by the inclusion $C_{1} \subset \mathscr{F} \cap C(X)$, we see that $\mathscr{F} \cap C(X)$ includes the countable collection $C_{1}^{\prime}$ which appeared in (3.6). The members of $C_{1}^{\prime}$ will be numbered as $g_{1}, g_{2}, \ldots, g_{k}, \ldots$ Put $X_{\alpha, n}^{k}=\left\{x ; g_{k}(x)-\alpha R_{\alpha+\alpha_{0}} g_{k}(x)>1 / n\right\}$, which includes the set $\left\{x ; g_{k}(x)>\int_{X} g_{k}(y) \mu(x, d y)+1 / n\right\}$ for every $\alpha>0$. Lemma 2.1(iii) of [10] and the estimate (1.6) lead us to

$$
\operatorname{Cap}\left(X_{\alpha, n}^{k}\right) \leqq n^{2} \mathscr{E}^{\alpha} 0\left(g_{k}-\alpha R_{\alpha+\alpha_{0}} g_{k}, g_{k}-\alpha R_{\alpha+\alpha_{0}} g_{k}\right) \rightarrow 0, \quad \alpha \rightarrow+\infty .
$$

For any $\varepsilon>0$, take $\varepsilon_{k}>0$ such as $\sum_{k=1}^{+\infty} \varepsilon_{k}<\varepsilon$. For each $k$ and $n$, choose $\alpha$ such that the open set $Y_{n}^{k}=X_{\alpha, n}^{k}$ has the capacity less than $\varepsilon_{k} / 2^{n+1}$. Now $X_{b}$ is included in the open set $\bigcup_{k} \bigcup_{n} Y_{n}^{k}$ whose capacity is less than $\varepsilon$, as was to be proved.

3.3. Symmetry of the process. Here we will observe how the behaviours of the associated Ray process reflect the symmetry of our Ray resolvent. It is clear that the symmetry of $\left\{R_{\alpha}(x, E), \alpha>0\right\}$ implies the symmetry of the associated transition function $\left\{P_{t}(x, E), t>0\right\}$ : for any $t>0$ and nonnegative Borel measurable functions $f$ and $g$ on $X$

$$
\int_{X} P_{t} f(x) \cdot g(x) m(d x)=\int_{X} f(x) \cdot P_{t} g(x) m(d x) \leqq+\infty .
$$

LEMMA 3.2. For $0<t_{1}<\cdots<t_{n-1}<t_{n}$ and nonnegative Borel measurable functions $f_{0}, f_{1}, \ldots, f_{n-1}, f_{n}$ on $X$,

$$
\begin{aligned}
\int_{X} E_{x}\left(f_{0}\left(X_{0}\right) f_{1}\left(X_{t_{1}}\right) \cdots f_{n-1}\left(X_{t_{n-1}}\right) f_{n}\left(X_{t_{n}}\right)\right) m(d x) \\
=\int_{X} E_{x}\left(f_{n}\left(X_{0}\right) f_{n-1}\left(X_{t_{n}-t_{n-1}}\right) \cdots f_{1}\left(X_{t_{n}-t_{1}}\right) f_{0}\left(X_{t_{n}}\right)\right) m(d x) .
\end{aligned}
$$

Proof. Notice that $P_{x}\left(X_{0}=x\right)=1$ for $m$-a.e. $x \in X$ because of (M.1) and Theorem 3.2 of the preceding subsection and Theorem 1.2(i). We will prove this lemma by induction. Suppose that (3.12) holds for a given $n$. Then

$$
\begin{aligned}
\int_{X} E_{x}\left(f_{0}\left(X_{0}\right) \cdots f_{n}\left(X_{t_{n}}\right) f_{n+1}\left(X_{t_{n+1}}\right)\right) m(d x) \\
\quad=\int_{X} E_{x}\left(f_{0}\left(X_{0}\right) \cdots\left(f_{n} \cdot P_{t_{n+1}-t_{n}} f_{n+1}\right)\left(X_{t_{n}}\right)\right) m(d x) \\
=\int_{X} P_{t_{n+1}-t_{n}} f_{n+1}(x) E_{x}\left(f_{n}\left(X_{0}\right) f_{n-1}\left(X_{t_{n}-t_{n-1}}\right) \cdots f_{0}\left(X_{t_{n}}\right)\right) m(d x)
\end{aligned}
$$

which is equal to

$$
\begin{array}{r}
\int_{X} f_{n+1}(x) P_{t_{n+1}-t_{n}}\left(E \cdot\left(f_{n}\left(X_{0}\right) f_{n-1}\left(X_{t_{n}-t_{n-1}}\right) \cdots f_{0}\left(X_{t_{n}}\right)\right)\right)(x) m(d x) \\
=\int_{X} E_{x}\left(f_{n+1}\left(X_{0}\right) f_{n}\left(X_{t_{n+1}-t_{n}}\right) \cdots f_{0}\left(X_{t_{n+1}}\right)\right) m(d x)
\end{array}
$$


by virtue of (3.11) and the Markov property. Thus (3.12) holds for $n+1$, completing the proof of Lemma 3.2.

Since $\partial$ is not a branch point of $M,(\mathbf{M} .3)$ implies as in [2, (9.3)] that the left limits of sample paths must lie in $X$ up to their lifetimes almost surely. In the following we assume without loss of generality that every $\omega \in W$ has the property that $X_{t-}(\omega) \in X$ for every $t<\zeta(\omega)$.

Fix a positive number $c>0$. Denote by $\mathfrak{R}$ the set of all functions $\varphi(t)(0 \leqq t \leqq c)$ taking values in $X$. The (time reversal) transformation $q$ of the space $\mathfrak{L}$ is defined by $q \varphi(t)=\varphi(c-t), 0 \leqq t \leqq c$. For $\omega \in W$ such as $X_{c-}(\omega) \in X$, we define $\nu_{r} \omega$ and $\nu_{l} \omega$ $\in \mathfrak{L}$ by

$$
\begin{aligned}
& \left(\nu_{r} \omega\right)(t)=X_{t}(\omega), \quad 0 \leqq t<c, \quad\left(\nu_{l} \omega\right)(t)=X_{0}(\omega), \quad t=0, \\
& =X_{c-}(\omega), t=c ; \quad=X_{t-}(\omega), \quad 0<t \leqq c .
\end{aligned}
$$

Finally we put for $\Gamma \subset\left\{X_{c-} \in X\right\}$,

$$
\gamma \Gamma=\nu_{l}^{-1} q \nu_{r} \Gamma\left({ }^{12}\right) .
$$

Denote by $\mathscr{B}_{(0, c)}$ the restriction to $\left\{X_{c-} \in X\right\}$ of the $\sigma$-field $\bigvee_{t<c} \mathscr{B}_{t}^{0}$.

THEOREM 3.3( $\left(^{13}\right)$. If $\Gamma \subset \mathscr{B}_{(0, c)}$, then $\gamma \Gamma \in \mathscr{B}_{(0, c)}$ and

$$
\int_{X} P_{x}(\gamma \Gamma) m(d x)=\int_{X} P_{x}(\Gamma) m(d x) \leqq+\infty .
$$

Proof. It suffices to prove the theorem for the set

$$
\Gamma=\left\{X_{0} \in E_{0}, X_{t_{1}} \in E_{1}, \ldots, X_{t_{n-1}} \in E_{n-1}, X_{c-} \in E_{n}\right\},
$$

where $0<t_{1}<t_{2}<\cdots<t_{n-1}<c$ and $E_{0}, \ldots, E_{n}$ are Borel subsets of $X$. Clearly

$$
\gamma \Gamma=\left\{X_{0} \in E_{n}, X_{\left(c-t_{n-1}\right)-} \in E_{n-1}, \ldots, X_{\left(c-t_{1}\right)-} \in E_{1}, X_{c-} \in E_{0}\right\} .
$$

By Lemma 3.2 we have for sufficiently small $\varepsilon>0$ and $\delta>0$,

$$
\begin{aligned}
\int_{X} E_{x}\left(f_{0}\left(X_{0}\right) f_{1}\left(X_{t_{1}+\varepsilon}\right) \cdots f_{n-1}\left(X_{t_{n}+\varepsilon}\right) f_{n}\left(X_{c-\delta}\right)\right) m(d x) \\
=\int_{X} E_{x}\left(f_{n}\left(X_{0}\right) f_{n-1}\left(X_{c-t_{n-1}-\varepsilon-\delta}\right) \cdots f_{1}\left(X_{c-t_{1}-\varepsilon-\delta}\right) f_{0}\left(X_{c-\delta}\right)\right) m(d x) .
\end{aligned}
$$

Assume that $f_{0}, f_{1}, \ldots, f_{n} \in C_{0}^{+}(X)$ and let $\varepsilon$ and $\delta$ tend to zero. Then after a routine procedure we get the equality (3.14) for $\Gamma$ of (3.15).

${ }^{(12)}$ The operator $\gamma$ was introduced by E. B. Dynkin [7, IV, \$4] in connection with the multidimensional Brownian motion. The present author used a similar notion in the analysis of a reflecting Brownian motion [8]. However the notion $\gamma$ defined in $[8$, p. 206] was insufficient for the situation there and he likes to correct it here: it must be replaced by the present definition (3.13).

(13) Cf. Theorem 4.12 of [7]. 
Here we give two applications of Theorem 3.3.

According to the proof of IV, T52 of P. A. Meyer [15] we observe that, for any Borel set $B \subset X$ and $t>0$, the set $\left\{\sigma_{B}^{\prime}<t\right\}$ is in the completion of the $\sigma$-field $\mathscr{B}_{t}^{0}$ relative to $P \mu, \mu$ being an arbitrary probability measure on $X$. This fact will be used in the proof of the following theorem:

THEOREM 3.4(14). For q.e. $x \in X$,

$$
P_{x}\left(X_{t-} \in X-X_{b} \text { for every } t \in(0, \zeta)\right)=1 .
$$

COROLLARY. If $T_{n}$ are increasing stopping times with limit $T$, then

$$
P_{x}\left(\lim _{n \rightarrow+\infty} X_{T_{n}}=X_{T}, T<\zeta\right)=P_{x}(T<\zeta)
$$

for q.e. $x \in X$.

This corollary is immediate from Theorem 3.4 and property (M.3). Here, the exceptional points $x \in X$ do not depend on the choice of $\left\{T_{n}\right\}$.

Proof of Theorem 3.4. Put $\Gamma_{c}=\left\{\sigma_{X_{b}}^{\prime}<c, X_{c-} \in X\right\}$ and $\Lambda_{c}=\left\{X_{t-} \in X_{b}\right.$ for some $\left.t \in(0, c), X_{c-} \in X\right\} . X_{b}$ is a Borel set (actually an $F_{\sigma}$-set) and $P_{x}\left(\Gamma_{c}\right)=0, x \in X$, according to (M.4). Hence there exists a set $\Gamma_{c}^{\prime} \in B_{(0, c)}$ such that $\Gamma_{c} \subset \Gamma_{c}^{\prime}$ and $\int_{X} P_{x}\left(\Gamma_{c}^{\prime}\right) m(d x)=0$. Since $\Lambda_{c}=\gamma \Gamma_{c} \subset \gamma \Gamma_{c}^{\prime}$, Theorem 3.3 implies that $P_{x}\left(\Lambda_{c}\right)=0$ for $m$-a.e. $x \in X$. Notice that $\Lambda=\left\{X_{t-} \in X_{b}\right.$, for some $\left.t \in(0, \zeta)\right\}=\bigcup_{c \in Q} \Lambda_{c}, Q$ being the set of all positive rational numbers. We have therefore $P_{x}(\Lambda)=0$ for $m$-a.e. $x \in X$. On the other hand $u(x)=P_{x}(\Lambda)$ is an excessive function: $u$ is universally measurable and

$$
\exp \left(-\alpha_{0} s\right) P_{s} u(x)=\exp \left(-\alpha_{0} s\right) P_{x}\left\{X_{t-} \in X_{b} \text { for some } t \in(s, \zeta)\right\} \uparrow u(x), \quad s \downarrow 0 .
$$

Thus $u(x)=0$ for q.e. $x \in X$ in view of Theorem 3.1 and Theorem 1.2.

Finally for an open or a closed set $A \subset X$ we define

$$
P_{t}^{0}(x, E)=P_{x}\left(X_{t} \in E, t<\sigma_{A}\right), \quad t \geqq 0 .
$$

$\left\{P_{t}^{0}(x, E), t \geqq 0\right\}$ is a sub-Markov transition probability on $X$. Since $P_{t}^{0} f(x)$ $=\int_{X} P_{t}^{0}(x, d y) f(y)$ is right continuous in $t \geqq 0$ for $f \in C_{0}(X), P_{t}^{0}(x, E)$ is measurable in $(t, x) \in[0,+\infty) \times X$ for each fixed Borel set $E \subset X$. Put

$$
R_{\alpha}^{0}(x, E)=\int_{0}^{+\infty} e^{-\alpha t} P_{t}^{0}(x, E) d t .
$$

$\left\{R_{\alpha}^{0}(x, E), \alpha>0\right\}$ is a sub-Markov resolvent on $X$. Obviously $R_{\alpha}^{0} f(x)=\int_{X} R_{\alpha}^{0}(x, d y) f(y)$ satisfies

$$
R_{\alpha}^{0} f(x)=E_{x}\left(\int_{0}^{\sigma_{A}} e^{-\alpha t} f\left(X_{t}\right) d t\right), \quad x \in X,
$$

if $f$ is Borel measurable and $R_{\alpha}^{0} f(x)$ is well defined.

(14) Cf. Lemma 3.7(iv) of [8]. 
THEOREM 3.5( $\left.{ }^{15}\right)$. For an open or a closed set $A \subset X$, the transition probability and the resolvent kernel defined by (3.18) and (3.19) are m-symmetric:

$$
\begin{aligned}
& \int_{X} f(x) \cdot P_{t}^{0} g(x) m(d x)=\int_{X} P_{t}^{0} f(x) \cdot g(x) m(d x), \quad t \geqq 0, \\
& \int_{X} f(x) \cdot R_{\alpha}^{0} g(x) m(d x)=\int_{X} R_{\alpha}^{0} f(x) \cdot g(x) m(d x), \quad \alpha>0,
\end{aligned}
$$

for $f, g \in C^{+}(X)$.

Proof. (3.22) is a direct consequence of (3.21). Let us show (3.21) when $A$ is an open set. Fix a positive number $c$ and Borel sets $F, G \subset X$. Consider the set $\Gamma=\left\{X_{0} \in F, c \leqq \sigma_{A}, X_{c-} \in G\right\}$. Since $A$ is open, $\Gamma \in \mathscr{B}_{(0, c)}$ and

$$
\gamma \Gamma=\left\{X_{0} \in G, c \leqq \sigma_{A}, X_{c-} \in F\right\} .
$$

Noting that $P_{x}\left(X_{0}=x\right)=1 m$-a.e., we get by Theorem 3.3,

$$
\int_{F} P_{x}\left(X_{c-} \in G, c \leqq \sigma_{A}\right) m(d x)=\int_{G} P_{x}\left(X_{c-} \in F, c \leqq \sigma_{A}\right) m(d x)
$$

from which follows the equality

$$
\int_{X} f(x) E_{x}\left(g\left(X_{c-}\right), c \leqq \sigma_{A}\right) m(d x)=\int_{X} E_{x}\left(f\left(X_{c_{-}}\right), c \leqq \sigma_{A}\right) g(x) m(d x)
$$

for $f, g \in C_{0}(X)$. By putting $c=t+1 / n$ and letting $n$ tend to infinity in this equality, we obtain (3.21).

Next suppose that $A$ is closed and choose a sequence of open sets $A_{n}$ such as $A_{n} \supset \bar{A}_{n+1} \supset A$ and $A_{n} \downarrow A$. By virtue of the quasi-left continuity (Corollary to Theorem 3.4)

$$
P_{x}\left(\sigma_{A_{n}} \uparrow \sigma_{A}\right)=1, \quad \text { q.e. } x \in X .
$$

Hence (3.21) for the closed set $A$ follows from those for open sets $A_{n}$.

3.4. Probabilistic decomposition of $\left(\mathscr{F}^{*}, \mathscr{E}^{\alpha}\right)$. Let $A$ be an open or a closed set of $X$. We put

$$
\begin{aligned}
& H_{\alpha}(x, E)=E_{x}\left(e^{-\alpha \sigma_{A}} ; X \sigma_{A} \in E\right), \\
& H_{\alpha}^{\prime}(x, E)=E_{x}\left(e^{-\alpha \sigma_{A}^{\prime}} ; X \sigma_{A}^{\prime} \in E\right) .
\end{aligned}
$$

In this subsection we will consider the kernels $H_{\alpha}$ and $H_{\alpha}^{\prime}$ on $X$ as well as the localized resolvent $R_{\alpha}^{0}$ defined by (3.19) and reveal the roles they play in the strongly regular $D$-space $(\mathscr{F}, \mathscr{E})$.

Any Borel measurable function on $X$ is extended to $\bar{X}=X \cup \partial$ by defining its value at $\partial$ to be zero. It holds under this convention that $H_{\alpha} f(x)=E_{x}\left(e^{-\alpha \sigma_{A}} f\left(X_{\sigma_{A}}\right)\right)$ and $R_{\alpha} f(x)=E_{x}\left(\int_{0}^{\xi} e^{-\alpha t} f\left(X_{t}\right) d t\right)$ for all $x \in \bar{X}$ when $f$ is Borel measurable and

${ }^{(15)}$ Cf. Lemma 14.1 of [7]. 
$H_{\alpha} f(x)=\int_{X} H_{\alpha}(x, d y) f(y)$ and $R_{\alpha} f(x)$ are well defined for $x \in X . H_{\alpha}^{\prime} f$ can be expressed in a similar way. If a Borel measurable function $f$ is excessive, then $H_{\alpha_{0}} f$ is supermedian and $H_{\alpha_{0}}^{\prime} f$ is excessive. When $A$ is open, $\sigma_{A}=\sigma_{A}^{\prime}$ and $H_{\alpha}=H_{\alpha}^{\prime}$.

LEMMA 3.3. If $f$ is a bounded Borel measurable function on $X$, then for each $x \in X$

$$
\begin{gathered}
R_{\alpha} f(x)=R_{\alpha}^{0} f(x)+H_{\alpha} R_{\alpha} f(x), \quad \alpha>0, \\
H_{\alpha} f(x)-H_{\beta} f(x)+(\alpha-\beta) R_{\alpha}^{0} H_{\beta} f(x)=0, \quad \beta>0 .
\end{gathered}
$$

Proof. We can use the strong Markov property (M.2) to obtain the formula (3.25). (3.26) is a result of the Markov property.

By virtue of Theorem 3.5, the kernel $\left\{R_{\alpha}^{0}(x, E), \alpha>0\right\}$ is an $m$-symmetric subMarkov resolvent kernel on $X$. Let $\left(\mathscr{F}^{(0)}, \mathscr{E}^{(0)}\right)$ be the Dirichlet space generated by $\left\{R_{\alpha}^{0}(x, E), \alpha>0\right\}: R_{\alpha}^{0}\left(L^{2}(X ; m) \cap C(X)\right) \subset \mathscr{F}^{(0)}$ and the function $R_{\alpha}^{0} f, f \in L^{2}(X ; m)$ $\cap C(X)$, satisfies

$$
\mathscr{E}^{(0), \alpha}\left(R_{\alpha}^{0} f, v\right)=(f, v)_{X} \quad \text { for every } v \in \mathscr{F}^{(0)} .
$$

THEOREM 3.6. $\mathscr{F}^{(0)} \subset \mathscr{F}$ and $\mathscr{E}^{(0)}$ is the restriction of $\mathscr{E}$ to $\mathscr{F}^{(0)}$ :

$$
\mathscr{E}^{(0)}(u, v)=\mathscr{E}(u, v), \quad u, v \in \mathscr{F}^{(0)}
$$

Proof. Denote by $\mathfrak{B}$ the set of all bounded Borel measurable functions on $X$. Since $R_{\alpha_{0}}^{0}\left(L^{2} \cap C\right)$ is dense in $\mathscr{F}^{(0)}$ with metric $\mathscr{E}^{(0), \alpha_{0}}$, it suffices to prove the following:

$$
\begin{aligned}
& \text { For every } f \in \mathfrak{B} \cap L^{2}, \quad R_{\alpha_{0}}^{0} f \in \mathscr{F} \text { and } \\
& \mathscr{E} \alpha_{0}\left(R_{\alpha_{0}}^{0} f, R_{\alpha_{0}}^{0} f\right)=\left(f, R_{\alpha_{0}}^{0} f\right)_{X} .
\end{aligned}
$$

We can observe by Lemma 1 of [9] that $u \in L^{2}(X ; m)$ is an element of $\mathscr{F}$ if and only if $\lim _{\beta \rightarrow+\infty} \beta\left(u-\beta R_{\beta+\alpha_{0}} u, u\right)_{X}$ is finite and in this case the limit is equal to $\mathscr{E}_{0}(u, u)$. Thus the relation (3.29) is equivalent to

$$
\lim _{\beta \rightarrow+\infty} \beta\left(u-\beta R_{\beta+\alpha_{0}} u, u\right)_{X}=(f, u)_{X}
$$

where $u=R_{\alpha_{0}}^{0} f, f \in \mathfrak{B} \cap L^{2}$.

Let us show (3.30) for an open set $A$. By (3.25) and the resolvent equation for $R_{\alpha}^{0}$,

$$
\beta\left(u-\beta R_{\beta+\alpha_{0}} u, u\right)_{X}=\beta\left(R_{\beta+\alpha_{0}}^{0} f, u\right)_{X}-\beta^{2}\left(H_{\beta+\alpha_{0}} R_{\beta+\alpha_{0}} u, u\right)_{X} .
$$

Since $R_{\alpha}^{0}$ is symmetric and its $L^{2}$-norm is no greater than $1 / \alpha$,

$$
\beta\left(R_{\beta+\alpha_{0}}^{0} f, u\right)_{X}=\left(f, R_{\alpha_{0}}^{0} f-R_{\beta+\alpha_{0}}^{0} f\right)_{X} \rightarrow(f, u)_{X}, \quad \beta \rightarrow+\infty .
$$

We have to prove

$$
\beta^{2}\left(H_{\beta+\alpha_{0}} R_{\beta+\alpha_{0}} u, u\right)_{X} \rightarrow 0, \quad \beta \rightarrow+\infty .
$$

We may assume without loss of generality that $f$ is nonnegative. By the symmetry of $R_{\alpha_{0}}^{0}$ and the formula (3.26), the left-hand side of (3.31) is no greater than 
$\beta\left(H_{\alpha_{0}} R_{\beta+\alpha_{0}} u, f\right)_{X}$. Notice that $u$ is the difference of two excessive functions: $u=R_{\alpha_{0}} f-H_{\alpha_{0}}\left(R_{\alpha_{0}} f\right)$. Hence $\lim _{\beta \rightarrow+\infty} \beta\left(H_{\alpha_{0}} R_{\beta+\alpha_{0}} u, f\right)_{X}=\left(H_{\alpha_{0}} u, f\right)_{X}$. However, since $\sigma_{A}\left(\theta_{\sigma_{A}} \omega\right)=0$ for $\omega \in\left\{\sigma_{A}<\zeta\right\}$, we have $H_{\alpha_{0}} \cdot H_{\alpha_{0}}\left(R_{\alpha_{0}} f\right)(x)=H_{\alpha_{0}}\left(R_{\alpha_{0}} f\right)(x), x \in X$, which means $H_{\alpha_{0}} u(x)=0, x \in X$, yielding (3.31). Here $\theta$ denotes the usual translation operator on $W$ (cf. [16]).

Thus (3.30) and hence (3.29) are established when $A$ is open. (3.29) for a closed set $A$ is now to be proved. Find open sets $A_{n}$ such that $A_{n} \supset \bar{A}_{n+1}$ and $A_{n} \downarrow A$. Denote by ${ }^{n} R_{\alpha}^{0}$ and ${ }^{n} H_{\alpha}$ kernels corresponding to $A_{n}$. Put $u_{n}={ }^{n} R_{\alpha_{0}}^{0} f$ for a nonnegative $f \in \mathfrak{B} \cap L^{2}$. Then owing to (3.20) and (3.23), $u_{n}$ increases to $R_{\alpha_{0}}^{0} f$ q.e. as $n \rightarrow+\infty$. Now observe the following equality: for $m \leqq n$,

$$
\begin{aligned}
& \beta\left(u_{n}-\beta R_{\beta+\alpha_{0}} u_{n}, u_{m}\right)_{X} \\
& \quad=\beta\left({ }^{m} R_{\beta+\alpha_{0}}^{0} f, u_{m}\right)_{X}+\beta\left({ }^{m} H_{\beta+\alpha_{0}}{ }^{n} R_{\beta+\alpha_{0}}^{0} f, u_{m}\right)_{X}-\beta^{2}\left({ }^{n} H_{\beta+\alpha_{0}} R_{\beta+\alpha_{0}} u_{n}, u_{m}\right)_{X} .
\end{aligned}
$$

Here we used the identity ${ }^{n} R_{\beta}^{0} f={ }^{m} R_{\beta}^{0} f+{ }^{m} H_{\beta}{ }^{n} R_{\beta}^{0} f$. Let $\beta$ tend to infinity. Then the first term of the right-hand side of the equality tends to $\left(f, u_{m}\right)_{X}$ as was proved earlier. The second term is, in view of the symmetry of ${ }^{m} R_{\alpha_{0}}^{0}$ and (3.26), no greater than $\left({ }^{m} H_{\alpha_{0}}{ }^{n} R_{\beta}^{0}+\alpha_{0} f, f\right)_{X}$, which decreases to zero. The absolute value of the last term is no greater than $\beta^{2}\left({ }^{n} H_{\beta+\alpha_{0}} R_{\beta+\alpha_{0}} u_{n}, u_{n}\right)_{X}$, which also tends to zero by (3.31). What we have proved is $\mathscr{E} \alpha_{0}\left(u_{n}, u_{m}\right)=\left(f, u_{m}\right)_{X}, m \leqq n$, which in turn tells us that $u_{n}$ converges to $R_{\alpha_{0}}^{0} f$ in $\mathscr{E}^{\alpha_{0}}$-norm, arriving at (3.29) for the closed set $A$. The proof of Theorem 3.6 is complete.

On account of Theorem $3.6, \mathscr{F}^{(0)}$ is a closed subspace of $\mathscr{F}$ with metric $\mathscr{E}^{\alpha_{0}}$. Let us denote by $\mathscr{H}_{\alpha_{0}}$ the orthogonal complement of $\mathscr{F}^{(0)}$ in the Hilbert space $\left(\mathscr{F}, \mathscr{E}^{\alpha}\right)_{0}$.

LEMMA $3.4\left({ }^{16}\right)$. If $u$ is either an element of $\mathscr{F} \cap C(X)$ or of the form $R_{\alpha_{0}} h$, $h \in \mathfrak{B} \cap L^{2}$, then $H_{\alpha_{0}} u$ is quasi-continuous and

$$
H_{\alpha_{0}} u=P \mathscr{H}_{\alpha_{0}} u
$$

$P \mathscr{H}_{\alpha_{0}}$ being the projection on the space $\mathscr{H}_{\alpha_{0}}$.

Proof. Let us first show this for $u=R_{\alpha_{0}} h, h \in \mathfrak{B} \cap L^{2}$. By (3.25), (3.29) and Lemma 3.1, we have

$$
\begin{aligned}
\mathscr{E}^{\alpha}{ }_{0}\left(H_{\alpha_{0}} R_{\alpha_{0}} h, R_{\alpha_{0}}^{0} g\right) & =\mathscr{E}^{\alpha \alpha_{0}}\left(R_{\alpha_{0}} h-R_{\alpha_{0}}^{0} h, R_{\alpha_{0}}^{0} g\right) \\
& =\left(h, R_{\alpha_{0}}^{0} g\right)_{X}-\left(h, R_{\alpha_{0}}^{0} g\right)_{X}=0
\end{aligned}
$$

for every $g \in \mathfrak{B} \cap L^{2}$. This means that the formula (3.25) represents the direct decomposition of $u=R_{\alpha_{0}} h$ into the sum of elements of $\mathscr{F}^{(0)}$ and $\mathscr{H}_{\alpha_{0}}$, getting (3.32). The quasi-continuity of $H_{\alpha_{0}} u$ is clear if $A$ is open, because it is then the difference

${ }^{(16)}$ We have further $H_{\alpha_{0}} u=H_{\alpha_{0}^{\prime}} u$, q.e. for $u \in \mathscr{F} \cap C(X)$. Since $H_{\alpha_{0}^{\prime}} R_{\alpha_{0}} u$ is the regularization of the quasi-continuous function $H_{\alpha_{0}} R_{\alpha_{0}} u$, these two are equal q.e. From this we can get the desired equality. 
of excessive functions $H_{\alpha_{0}}\left(R_{\alpha_{0}} h^{+}\right)$and $H_{\alpha_{0}}\left(R_{\alpha_{0}} h^{-}\right)$of $\mathscr{F}$ to which we can apply Theorem 3.1. Coming to the case when $A$ is closed, consider open sets $A_{n}$ and corresponding kernels ${ }^{n} R_{\alpha_{0}}^{0}$ and ${ }^{n} H_{\alpha_{0}}$ just as in the second part of the proof of Theorem 3.6. Then the quasi-continuous functions ${ }^{n} H_{\alpha_{0}} u=u-{ }^{n} R_{\alpha_{0}}^{0} h$ converge to $u-R_{\alpha_{0}}^{0} h=H_{\alpha_{0}} u$ q.e. on $X$ as well as in $\mathscr{E}^{\alpha_{0}}$-norm. Hence the latter must be quasicontinuous (Lemma 1.2).

Next take any $u \in \mathscr{F} \cap C(X)$. Since, for each $\beta>0, R_{\beta} u$ is equal to $R_{\alpha_{0}} h$ with some $h \in \mathfrak{B} \cap L^{2}$, we have $H_{\alpha_{0}}\left(\beta R_{\beta} u\right)=P \mathscr{H}_{\alpha_{0}}\left(\beta R_{\beta} u\right)$.

By Lemma 2.1 of [10], $\beta R_{\beta} u \rightarrow u$ and hence

$$
P_{\mathscr{K}_{\alpha_{0}}}\left(\beta R_{\beta} u\right) \rightarrow P_{\mathscr{H}_{\alpha_{0}}} u, \quad \beta \rightarrow+\infty,
$$

with respect to $\mathscr{E}^{\alpha_{0}}$-norm. On the other hand,

$$
\begin{aligned}
\lim _{\beta \rightarrow+\infty} H_{\alpha_{0}}\left(\beta R_{\beta} u\right)(x) & =\lim _{\beta \rightarrow+\infty} E_{x}\left(\exp \left(-\alpha_{0} \sigma_{A}\right) \beta G_{\beta} u\left(X_{\sigma_{A}}\right) ; X_{\sigma_{A}} \notin X_{b}\right) \\
& =E_{x}\left(\exp \left(-\alpha_{0} \sigma_{A}\right) u\left(X_{\sigma_{A}}\right)\right)=H_{\alpha_{0}} u(x), \quad x \in X,
\end{aligned}
$$

by virtue of property (M.4). Thus we get (3.32). $H_{\alpha_{0}} u$ is quasi-continuous because it is the limit of quasi-continuous functions $H_{\alpha_{0}}\left(\beta R_{\beta} u\right)$ in $\mathscr{E}^{\alpha_{0}}$-norm as well as in the pointwise sense.

LEMMA 3.5. Suppose that $A$ is compact. Any quasi-supermedian function belonging to the space $\mathscr{H}_{\alpha_{0}}$ is a potential of a measure whose support is concentrated on $A$.

Proof. Assume that $u$ is quasi-supermedian and $u \in \mathscr{H}_{\alpha_{0}}$. Then $u \in \mathscr{F}^{*}$ and we have by Lemma 1.3 that $\mathscr{E}^{\alpha} 0(u, v) \geqq 0$ for all $v \in \mathscr{F}^{*}$ such as $v \geqq 0$ q.e. Let $v$ be any function of $\mathscr{F} \cap C(X)$ which is nonnegative on $A$. By Lemma 3.4, $\mathscr{E}^{\alpha} \alpha_{0}(u, v)$ $=\mathscr{E} \alpha_{0}\left(u, H_{\alpha_{0}} v\right)$ which is nonnegative because $H_{\alpha_{0}} v(x) \geqq 0, x \in X$. According to Theorem 1.6, we arrive at Lemma 3.5.

The next two are the main theorems of this subsection.

THEOREM 3.7( $\left.{ }^{17}\right)$. Put

$$
\mathscr{F}_{X-A}^{*}=\left\{u \in \mathscr{F}^{*} ; u=0 \text { q.e. on } A\right\} .
$$

Then $\mathscr{F}_{X-A}^{*}=\left(\mathscr{F}^{(0)}\right)^{*}$, where $\left(\mathscr{F}^{(0)}\right)^{*}$ denotes the set of all quasi-continuous modifications of functions in the space $\mathscr{F}^{(0)}$.

Proof. On account of Theorem 3.6 and Lemma 1.2, $\left(\mathscr{F}^{(0)}\right)^{*}$ and $\mathscr{F}_{X-A}^{*}$ are closed subspaces of the Hilbert space $\left(\mathscr{F} *, \mathscr{E}^{\alpha_{0}}\right)$. If $f \in L^{2} \cap C$, then $R_{\alpha_{0}}^{0} f(x)=0$ on $A-X_{b}$ and $R_{\alpha_{0}}^{0} f=R_{\alpha_{0}}^{0} f-H_{\alpha_{0}}\left(R_{\alpha_{0}} f\right)$ is quasi-continuous in view of Lemma 3.4. Hence $\mathscr{F}_{X-A}^{*}$ contains $R_{\alpha_{0}}^{0}\left(L^{2} \cap C\right)$ which is dense in $\left(\mathscr{F}^{(0)}\right)^{*}$. Thus, $\mathscr{F}_{X-A}^{*} \supset\left(\mathscr{F}^{(0)}\right)^{*}$.

${ }^{\left({ }^{17}\right)}$ We can assert even more: for any nonnegative universally measurable function $f \in L^{2}(X ; m), R_{\alpha}^{0} f$ belongs to the space $\mathscr{F}_{X-A}^{*}$ and the equation $\mathscr{E}^{\alpha}\left(R_{\alpha}^{0} f, v\right)=(f, v)_{X}$ holds for every $v \in \mathscr{F}_{X-A}^{*}$. In view of the proof of Theorem 3.7, this is true for $f \in L^{2} \cap C(X)$. Now the general case can be obtained exactly in the same manner as in the proof of Lemma 3.1. 
Let us prove the converse inclusion. Denote by $\mathscr{H}_{\alpha_{0}}^{*}$ the space of all quasicontinuous modifications of functions of the $\mathscr{H}_{\alpha_{0}}$. It suffices to show that $\mathscr{H}_{\alpha_{0}}^{*}$-the orthogonal complement of $\left(\mathscr{F}^{(0)}\right)^{*}$ in $\left(\mathscr{F}^{*}, \mathscr{E}^{\alpha_{0}}\right)$-is orthogonal to $\mathscr{F}_{X-A}^{*}$. Since $H_{\alpha_{0}} R_{\alpha_{0}}\left(L^{2} \cap C\right)$ is in $\mathscr{H}_{\alpha_{0}}^{*}$ by Lemma 3.4 and dense there, it is enough to prove

$$
\mathscr{E}^{\alpha_{0}}\left(H_{\alpha_{0}} R_{\alpha_{0}} f, v\right)=0, \quad f \in L^{2} \cap C^{+}, v \in \mathscr{F}_{X-A}^{*} .
$$

Assume first that $A$ is compact. Since $H_{\alpha_{0}} R_{\alpha_{0}} f$ is supermedian, it is quasi-supermedian (Remark 3.1) and is a potential of a measure $\mu \in M_{0}^{+}$with $S \mu \subset A$ by virtue of Lemma 3.5. Owing to Theorem 1.5, the left-hand side of (3.34) is equal to $\int_{A} v(x) \mu(d x)=0$.

In the case when $A$ is open or closed, we can find compact sets $A_{n}$ such that $A_{n} \uparrow A$. Denote by ${ }^{n} H_{\alpha_{0}}$ the kernel corresponding to $A_{n}$. It is easy to see that ${ }^{n} H_{\alpha_{0}} R_{\alpha_{0}} f$ then converges to $H_{\alpha_{0}} R_{\alpha_{0}} f$ increasingly and in $\mathscr{E}^{\alpha_{0}}$-norm. If $v \in \mathscr{F}_{X-A}^{*}$, then $v \in \mathscr{F}_{X-A_{n}}^{*}$ and the left-hand side of (3.34) is equal to $\lim _{n} \mathscr{E}^{\alpha_{0}}\left({ }^{n} H_{\alpha_{0}} R_{\alpha_{0}} f, v\right)=0$. The proof of Theorem 3.7 is complete.

Owing to this theorem, we get an important conclusion about a local property of the space $\mathscr{H}_{\alpha_{0}}^{*}$ : Any function in $\mathscr{H}_{\alpha_{0}}^{*}$ is determined by its restriction to the set $A$. In fact the space $\left(\mathscr{F} *, \mathscr{E}^{\alpha_{0}}\right)$ is expressed as a direct sum $\mathscr{F}^{*}=\mathscr{F}_{X-A .}^{*} \oplus \mathscr{H}_{\alpha_{0}}^{*}$ and so any function of $\mathscr{H}_{\alpha_{0}}^{*}$ which vanishes q.e. on $A$ should vanish q.e. on $X$. Keeping this in mind let us prove the next theorem.

THEOREM 3.8. Suppose that $A$ is a compact set or an open set belonging to the class $\mathscr{U}$. Then we have for q.e. $x \in X$,

$$
e_{A}(x)=E_{x}\left(\exp \left(-\alpha_{0} \sigma_{A}\right) ; \sigma_{A}<\zeta\right)=E_{x}\left(\exp \left(-\alpha_{0} \sigma_{A}^{\prime}\right) ; \sigma_{A}^{\prime}<\zeta\right) .
$$

Here $e_{A}$ denotes the equilibrium potential of $A$ defined in subsection 1.5.

Proof. Suppose that $A$ is compact. By (1.25) and (1.26), $e_{A}$ is an element of $\mathscr{H}_{\alpha_{0}}^{*}$ which is equal to 1 q.e. on $A$. The function $u(x)=E_{x}\left(\exp \left(-\alpha_{0} \sigma_{A}\right) ; \sigma_{A}<\zeta\right)$ has the same property. Indeed $u(x)=1, x \in A-X_{b}$, and hence q.e. on $A$ by Theorem 3.2. $u$ can be expressed as $H_{\alpha_{0}} f(x)$ with any function $f \in \mathscr{F} \cap C(X)$ such as $f(x)=1$ for $x \in A$. Hence $u \in \mathscr{H}_{\alpha_{0}}^{*}$ by Lemma 3.4. Thus we get the first equality of (3.35). The third term of (3.35) is the regularization of the second. Hence they are equal q.e. on $X$ in view of Theorem 3.1 .

When $A$ is an open set of the class $\mathscr{U},(3.35)$ is also obtained by approximating $A$ with a sequence of compact sets increasing to $A$ and by noting (1.22) and (1.23).

3.5. Regularity of quasi-continuous transformations along sample paths. Let us begin with a lemma which states a probabilistic feature of polar sets.

LEMMA 3.6. Let $A$ be a Borel polar set and $\left\{A_{n}\right\}$ be a decreasing sequence of open sets such that $A_{n} \supset A$ and $\lim _{n \rightarrow+\infty} \operatorname{Cap}\left(A_{n}\right)=0$. Then the equalities

$$
P_{x}\left(X_{t} \text { or } X_{t-} \in A \text { for some } t \geqq 0\right)=0 \text {, }
$$




$$
P_{x}\left(\sigma_{A_{n}}=\zeta \text { for some } n \text { or } \lim _{n \rightarrow+\infty} \sigma_{A_{n}}=+\infty\right)=1
$$

hold for q.e. $x \in X$.

Proof. (3.36) is a consequence of (3.37). In order to show (3.37), let us put $u_{n}(x)=E_{x}\left(\exp \left(-\alpha_{0} \sigma_{A_{n}}\right) ; \sigma_{A_{n}}<\zeta\right)$. By Theorem 3.8 and (1.21) we have $\mathscr{E}^{\alpha_{0}}\left(u_{n}, u_{n}\right)$ $=\mathrm{Cap}\left(A_{n}\right)$ which decreases to zero by the assumption. Since $u_{n}$ is quasi-continuous, Lemma 1.2 implies that $\lim _{n \rightarrow+\infty} u_{n}(x)=0$ q.e. on $X$. We arrive at (3.37) on account of the identity

$$
\lim _{n \rightarrow+\infty} u_{n}(x)=E_{x}\left(\exp \left(-\alpha_{0} \lim _{n} \sigma_{A_{n}}\right) ; \bigcap_{n=1}^{+\infty}\left\{\sigma_{A_{n}}<\zeta\right\}\right) .
$$

Since the branch set $X_{b}$ is polar (Theorem 3.2), we can apply the above lemma to $X_{b}$ to get

$$
P_{x}\left(X_{t-} \in X_{b} \text { for some } t \geqq 0\right)=0 \text {, }
$$

for q.e. $x \in X$. Notice that (3.38) is stronger than (3.16). We will further strengthen the assertions of Lemma 3.6 as follows:

THEOREM 3.9. Under the same assumption as in Lemma 3.6, there exists a Borel polar set $B$ including $A$ such that the equalities (3.37) and

$$
P_{x}\left(X_{t} \text { or } X_{t-} \in B \text { for some } t \geqq 0\right)=0
$$

are simultaneously valid for every $x \in X-B$.

Proof. By virtue of Lemma 3.6, we see that (3.36) and (3.37) are valid for every $x \in X$ except on a polar set $N_{1}$. By replacing $N_{1}$ with a $G_{\delta}$-polar set including it if necessary, we may assume that $N_{1}$ is a Borel set. Apply again Lemma 3.6 to the Borel polar set $A \cup N_{1}$. We get

$$
P_{x}\left(X_{t} \text { or } X_{t-} \in A \cup N_{1} \text { for some } t \geqq 0\right)=0
$$

for every $x \in X$ except on a Borel polar set $N_{2}$. Repeating the same argument, we have a sequence $\left\{N_{k}\right\}$ of Borel polar sets such that for each $k$ the equality

$$
P_{x}\left(X_{t} \text { or } X_{t-} \in A \cup N_{1} \cup \cdots \cup N_{k} \text { for some } t \geqq 0\right)=0
$$

holds for every $x \in X-N_{k+1}$. Put $B=A \cup\left(\bigcup_{k=1}^{+\infty} N_{k}\right)$. $B$ is polar by Theorem 1.1. If $x \in X-B$, then (3.41) is valid for every $k$. Letting $k$ tend to infinity, we get (3.39).

Turning to the main task of this subsection, let us consider a quasi-continuous function $q$ on $X$ taking values in some nice topological space. We fix a decreasing sequence $\left\{A_{n}\right\}$ of open subsets of $X$ such that $q$ is continuous on each $X-A_{n}$ and 
$\lim _{n \rightarrow+\infty} \operatorname{Cap}\left(A_{n}\right)=0$. By virtue of Theorem 3.9, there is a Borel polar set $B$ such that $B \supset \bigcap_{n=1}^{+\infty} A_{n}$ and equalities (3.37) and (3.39) hold for every $x \in \bar{X}-B$ : if we put

$$
\begin{aligned}
& W_{11}=\left\{\omega \in W ; X_{t}(\omega) \text { and } X_{t-}(\omega) \in \bar{X}-B \text { for all } t \geqq 0\right\}, \\
& W_{12}=\left\{\omega \in W ; \sigma_{A_{n}}(\omega)=\zeta(\omega) \text { for some } n \text { or } \lim _{n \rightarrow+\infty} \sigma_{A_{n}}(\omega)=+\infty\right\},
\end{aligned}
$$

then

$$
P_{x}\left(W_{1}\right)=1, \quad x \in \bar{X}-B,
$$

where $W_{1}$ denotes the set $W_{11} \cap W_{12}$.

Now let us put

$$
\mathscr{B}^{1}=\mathscr{B} \cdot W_{1}, \quad \mathscr{B}_{t}^{1}=\mathscr{B}_{t} \cdot W_{1}, \quad t \geqq 0,
$$

and denote the restrictions of measures $P_{x}, x \in \bar{X}-B$, to $\mathscr{B}^{1}$ by $P_{x}$ again. We also maintain the notion $X_{t}$ to express its restriction to $W_{1}$. It is then clear that the process $M_{1}=\left\{W_{1}, \mathscr{B}^{1}, \mathscr{B}_{t}^{1}, X_{t}, P_{x}\right\}$ is a right continuous Markov process with state space $\bar{X}-B$.

THEOREM 3.10. (i) The process $M_{1}$ is a strong Markov process with state space $\bar{X}-B$. The resolvent kernel of $M_{1}$ is the restriction to $X-B$ of the Ray resolvent kernel $\left\{R_{\alpha}(x, E), \alpha>0\right\}$ of the original process $M$. Further

$$
R_{\alpha}(x, B)=0, \quad x \in X-B .
$$

(ii) The o-field $\mathscr{B}^{1}$ (resp. $\mathscr{B}_{t}^{1}$ ) is the completion of $\mathscr{B}^{0} \cdot W_{1}\left(\right.$ resp. $\mathscr{B}_{t}^{0} \cdot W_{1}$ in $\mathscr{B}^{1}$ ) with respect to the family of measures

$$
\left\{P_{\mu}(\cdot)=\int_{\bar{X}-B} \mu(d x) P_{x}(\cdot) ; \mu \text { is a finite measure on } \bar{X}-B\right\} .
$$

(iii) Assume an additional condition that

$$
X_{b} \subset \bigcap_{n=1}^{+\infty} A_{n}
$$

Then the process $\boldsymbol{M}_{1}$ is quasi-left continuous on $[0,+\infty)$ : if $\left\{\mathscr{B}_{t}^{1}\right\}$-stopping times $T_{n}$ increase to $T$, then $\lim _{n \rightarrow+\infty} X_{T_{n}}=X_{T} P_{x}$-a.e. on $\{T<+\infty\}$ for every $x \in \bar{X}-B$.

(iv) Assume an additional condition that

(3.47) $q$ can be extended to $\bar{X}-\bigcap_{n=1}^{+\infty} A_{n}$ in such a way that the restriction of $q$ to $\bar{X}-A_{n}$ is continuous there for every $n$.

Then, for each $\omega \in W, q\left(X_{t}(\omega)\right)$ and $q\left(X_{t-}(\omega)\right)$ are well defined and $Y_{t}(\omega)=q\left(X_{t}(\omega)\right)$ is right continuous in $t \geqq 0$. $Y_{t}(\omega)$ has the left limit at every $t>0$ with

$$
Y_{t-}(\omega)=q\left(X_{t-}(\omega)\right) \text {. }
$$

(v) Assume that both the conditions (3.46) and (3.47) are valid. If $\left\{\mathscr{B}_{t}^{1}\right\}$-stopping times $T_{n}$ increase to $T$, then $\lim _{n \rightarrow+\infty} Y_{T_{n}}=Y_{T} P_{x}$-a.e. on $\{T<+\infty\}$ for every $x \in \bar{X}-B$. 
Proof. (i) The latter assertion together with (3.45) is evident. $M_{1}$ is now a Markov process on $\bar{X}-B$ with right continuous sample paths and right continuous $\sigma$-fields $\left\{\mathscr{B}_{t}^{1}\right\}$. Obviously the right continuity of $R_{\alpha} f, f \in C(X)$, along the sample paths is preserved under the transfer from $\boldsymbol{M}$ to $\boldsymbol{M}_{1}$. Thus, $\boldsymbol{M}_{1}$ is a strong Markov process on $\bar{X}-B$.

(ii) Take a set $\Lambda \in \mathscr{B}^{1}$ and consider its property with respect to the original process $\boldsymbol{M}$. Property (M.1) implies that

$$
P_{x}(\Lambda) \leqq P_{x}\left(W_{11} \cdot\left\{X_{0}=x\right\}\right)=0 \text { if } x \in B-X_{b}
$$

and

$$
P_{x}(\Lambda)=\int_{\bar{X}-X_{b}} \mu(x, d y) P_{y}(\Lambda)=\int_{\bar{X}-B} \mu(x, d y) P_{y}(\Lambda) \quad \text { if } x \in X_{b} .
$$

Therefore, for any finite measure $\mu$ on $\bar{X}, P_{\mu}(\Lambda)=P_{\mu_{1}}(\Lambda)$ with a finite measure $\mu_{1}$ supported by the set $\bar{X}-B$. This means statement (ii).

(iii) By the hypothesis (3.46),

$$
P_{x}\left(X_{t-} \in X_{b} \text { for some } t \geqq 0\right)=0, \quad x \in \bar{X}-B \text {. }
$$

Combining this with statement (i), we can prove the quasi-left continuity of $\boldsymbol{M}_{1}$ on $[0,+\infty)$ exactly in the same way as in $[13, \S 2]$ (see [16, XIV, T15] for more information).

(iv) Fix an $\omega \in W_{1}$. If $\zeta(\omega)<+\infty$, then $\sigma_{A_{n}}(\omega)=\zeta(\omega)$ for some $n$ and hence $X_{t}(\omega)$ and $X_{t-}(\omega)$ belong to the closed set $\bar{X}-A_{n}$ for all $t \geqq 0$. Hence we get the desired properties of $Y_{t}$ by the hypothesis (3.47). If $\zeta(\omega)=+\infty$, then for any $t \geqq 0$ there exists an $A_{n}$ such that $\sigma_{A_{n}}(\omega)>t$. Hence we get the desired conclusion in this case also.

(v) By the preceding two statements (iii) and (iv), we have $\lim _{n \rightarrow+\infty} Y_{T_{n}}$ $=q\left(\lim _{n \rightarrow+\infty} X_{T_{n}}\right)=q\left(X_{T}\right)=Y_{T} P_{x}$-a.e. on $\{T<+\infty\}$ for every $x \in \bar{X}-B$. The proof of Theorem 3.10 is complete.

REMARK 3.2. Here we give some remarks on the hypotheses (3.46) and (3.47) in Theorem 3.10. We can assume (3.46) without loss of generality because the branch set $X_{b}$ is polar. Assertions (iv) and (v) are still valid up to the lifetime $\zeta$ without assuming (3.47). Condition (3.47) is satisfied by two important cases in which we have interest. Theorem 1.3 implies that each numerical function $u \in \mathscr{F}$ has a modification $\tilde{u}$ which is not only quasi-continuous but also satisfies (3.47) by setting $\tilde{u}(\partial)=0$. In case that $q$ is a quasi-homeomorphism from $X$ to the underlying space $X^{\prime}$ of some regular $D$-space, $q$ satisfies $(3.47)$ if we put $q(\partial)=\partial^{\prime}$. We can see this immediately from the definition of quasi-homeomorphism.

Theorem 3.10 will be the key to prove Theorem 4.1. Here we state another application of Theorem 3.10. Consider a function $u$ defined q.e. on $X$. Let us agree to say $u$ to be Borel (resp. universally) measurable if there is a Borel (resp. universally) measurable function $\tilde{u}$ on $X$ such as $u=\tilde{u}$ q.e. We call $u$ finely continuous q.e. if 
there exists a nearly Borel polar set $B$ satisfying the following: $B \supset X_{b}, X-B$ is a fine open set and $u$ is finely continuous at each point $x \in X-B$, fine topology being defined in terms of $\boldsymbol{M}\left({ }^{18}\right)$. For instance take a quasi-continuous function $u$ (not necessarily real valued). Then $u$ is clearly Borel measurable in the above sense. Furthermore from the first and second remarks in Remark 3.2, we can see that $u$ is finely continuous q.e. Thus we get the first part of the following theorem.

THEOREM 3.11. (i) Every quasi-continuous function on $X$ is finely continuous q.e. and Borel measurable.

(ii) Conversely if a function $u$ of $\mathscr{F}$ is finely continuous q.e. and Borel (or more generally, universally) measurable, then $u$ is quasi-continuous.

Suppose that a function $u \in \mathscr{F}$ is finely continuous q.e. and universally measurable. Denote by $\tilde{u}$ a quasi-continuous modification of $u$. Then by the first part of Theorem 3.11, the $m$-negligible function $v=u-\tilde{u}$ is finely continuous q.e. Therefore the second part of Theorem 3.11 follows from the next lemma which is a counterpart of Theorem 1.2(ii).

LEMMA 3.7. If a function $v$ is finely continuous q.e. and universally measurable and if $v=0$ m-a.e., then $v=0$ q.e.

Proof. By making use of Theorem 3.9, we see that there is a Borel polar set $B \supset X_{b}$ such that $X-B$ is finely open, $v$ is finely continuous at each point of $X-B$ and $v$ is universally measurable on $X-B$. The set $C=\{x \in X-B ; v(x) \neq 0\}$ is then a fine open and universally measurable set which is consequently contained in the set $D=\left\{x \in X-B ; R_{\alpha_{0}}(x, C)>0\right\}$. Since $C$ is $m$-negligible and $R_{\alpha_{0}}$ is symmetric, $D$ is $m$-negligible. Hence $D$ becomes polar by virtue of Theorem 1.2(ii) and Lemma 3.1. $v$ now vanishes except on the polar set $B \cup C$.

3.6. Polar sets and absolute continuity conditions. The first half of the preceding subsection gives us probabilistic interpretations of polar sets. Here we will complete them.

We say a Borel set $Y \subset X$ is $M$-invariant if the equality

$$
P_{x}\left(X_{t} \text { and } X_{t-} \in Y \cup \partial \text { for all } t \geqq 0\right)=1
$$

holds for every $x \in Y$.

THEOREM 3.12. The following statements are equivalent to each other:

(i) $A$ Borel set $A$ is polar.

(ii) For m-almost all $x \in X$,

$$
P_{x}\left(\sigma_{A}^{\prime}<\zeta\right)=0
$$

(iii) There exists an m-negligible Borel set $B$ including $A$ such that $X-B$ is M-invariant.

${ }^{(18)}$ See $[2, \mathrm{II}]$ or $[16, \mathrm{XV}]$. 
Proof. (i) implies (iii) according to Theorem 3.9. Statement (iii) means (ii). Suppose that statement (ii) is valid. Then $E_{x}\left(\exp \left(-\alpha_{0} \sigma_{K}^{\prime}\right) ; \sigma_{K}^{\prime}<\zeta\right)=0 m$-a.e., for any compact set $K \subset A$. We have Cap $(K)=0$ by Theorem 3.8 and (1.21). Owing to (1.5) we arrive at the statement (i).

It should be noticed that we cannot generally strengthen the above statement (ii) by replacing " $m$-almost all $x$ " with "all $x$ ". The simplest example illustrative of this point is the case when $\mathscr{F}=L^{2}(X)$ and $\mathscr{E} \equiv 0$. In this case each $m$-negligible set is polar but every point of $X$ is trap with respect to the corresponding Ray process. The next theorem will concern the conditions to eliminate such irregular situations.

Suppose that a Borel set $A$ is of potential zero: $R_{\alpha}(x, A)=0$ for all $x \in A$ and $\alpha>0$. Then $m(A)=0$. Indeed symmetry of the kernel implies $\alpha \int_{A} R_{\alpha} 1(x) m(d x)$ $=\alpha \int_{X} R_{\alpha}(x, A) m(d x)=0$. Letting $\alpha$ tend to infinity, we get $m(A)=m\left(A-X_{b}\right)=0$.

THEOREM 3.13. The following conditions are mutually equivalent:

(i) $A$ Borel set $A$ is polar if and only if (3.49) is satisfied for all $x \in X$.

(ii) $m$ is a reference measure of $M: a$ set $A$ is of potential zero if and only if $m(A)=0$.

(iii) $R_{\alpha}(x, \cdot)$ is absolutely continuous with respect to $m$ for each $x \in X$ and $\alpha>0$.

Proof. It suffices to show the equivalence of (i) and (iii). Suppose that condition (iii) is satisfied and consider a Borel polar set $A$. By Theorem 3.12, we have $u(x)$ $=P_{x}\left(\sigma_{A}^{\prime}<\zeta\right)=0, m$-a.e., and consequently $u(x)=\lim _{\beta \rightarrow+\infty} \beta R_{\beta} u(x)=0$ for every $x \in X$. Thus condition (i) is valid. Conversely assume that (i) is met. Let $A$ be an $m$-negligible Borel set. Symmetry of the kernel and Lemma 3.1 then imply that $R_{\alpha}(x, A)=0$ holds for every $x \in X$ except on a polar set, which is of potential zero under condition (i). Thus we have $R_{\alpha}(x, A)=\lim _{\beta \rightarrow+\infty} \beta R_{\beta+\alpha} R_{\alpha}(x, A)=0$ for every $x \in X$ arriving at condition (iii).

REMARK 3.3. Suppose that condition (iii) of Theorem 3.13 is satisfied. Theorem 3.13 then tells us that we can adopt the set $\left(\bigcap_{n=1}^{\infty} A_{n}\right) \cup X_{b}$ as the set $B$ in Theorems 3.9 and 3.10 .

4. Regular Dirichlet spaces and strong Markov processes. Let $(X, m, \mathscr{F}, \mathscr{E})$ be a regular $D$-space. We adjoin a point $\partial$ to $X$ as the point at infinity if $X$ is noncompact and as an isolated point if $X$ is compact.

4.1. Construction of a strong Markov process-proof of Theorem 4.1. This subsection is devoted to the proof of Theorem 4.1 mentioned in the beginning of the present paper.

(I) By Theorem 3 of [10], there exists a strongly regular $D$-space $(\tilde{X}, \tilde{m}, \mathscr{F}, \mathscr{E})$ which is equivalent to $(X, m, \mathscr{F}, \mathscr{E})$. Every notion related to this strongly regular $D$-space will be written with tilde $\sim$. We already have several related notions specified in $\S 3$ - the Ray resolvent $\left\{\tilde{R}_{\alpha}(\tilde{x}, \tilde{E}), \alpha>0\right\}$, the branch set $\tilde{X}_{b}$, the transition function $\left\{\widetilde{P}_{t}(\tilde{x}, \widetilde{E}), t>0\right\}$ and the Ray process $\left(\tilde{W}, \tilde{\mathscr{B}}_{t}, \widetilde{P}_{\tilde{x}}\right)$ on the extended space $\tilde{X} \cup \delta$. 
By Theorem 2.1, there exists a capacity preserving quasi-homeomorphism $q$ from $X$ to $\tilde{X}$ : there are decreasing sequences of open sets $A_{k} \subset X$ and $\tilde{A}_{k} \subset \tilde{X}$ such that

$$
\lim _{k \rightarrow+\infty} \operatorname{Cap}\left(A_{k}\right)=0, \quad \lim _{k \rightarrow+\infty} \operatorname{Cap}^{\sim}\left(\tilde{A}_{k}\right)=0
$$

and the restriction of $q$ to $X-A_{k}$ is homeomorphic onto $\tilde{X}-\tilde{A}_{k}$ for each $k$. The equality (2.1) holds for every analytic set $A \subset X-\bigcap_{k=1}^{+\infty} A_{k}$. If we extend $q$ by setting $q(\partial)=\tilde{\partial}$, then according to Remark 3.2 ,

(4.1) the restriction of $q$ to $X \cup \partial-A_{k}$ is a homeomorphism onto $\tilde{X} \cup \tilde{\partial}-\tilde{A}_{k}$. Moreover we can assume without loss of generality that

$$
\tilde{X}_{b} \subset \bigcap_{k=1}^{+\infty} \tilde{A}_{k}
$$

because we can replace $\tilde{A}_{k}\left(\right.$ resp. $\left.A_{k}\right)$ with

$$
\tilde{A}_{k} \cup \tilde{D}_{k}=\tilde{A}_{k} \cup\left(\tilde{D}_{k}-\bigcap_{n=1}^{+\infty} \tilde{A_{n}}\right) \quad\left(\operatorname{resp} . A_{k} \cup q^{-1}\left(\tilde{D}_{k}-\bigcap_{n=1}^{+\infty} \tilde{A}_{n}\right)\right)
$$

if necessary. Here $\left\{\tilde{D}_{k}\right\}$ is a decreasing sequence of open sets of $\tilde{X}$ such that $\widetilde{D}_{k} \supset \tilde{X}_{b}$ and $\lim _{k \rightarrow+\infty} \operatorname{Cap}^{\sim}\left(\widetilde{D}_{k}\right)=0$.

By Theorem 3.9, there exists a Borel polar set $\tilde{B} \supset \bigcap_{k=1}^{+\infty} \tilde{A_{k}}$ which satisfies the following: if we put

$$
\begin{aligned}
& \tilde{W}_{11}=\left\{\tilde{\omega} \in \tilde{W} ; \tilde{X}_{t}(\tilde{\omega}) \text { and } \tilde{X}_{t-}(\tilde{\omega}) \in \tilde{X} \cup \tilde{\partial}-\tilde{B} \text { for all } t \geqq 0\right\}, \\
& \tilde{W}_{12}=\left\{\tilde{\omega} \in \tilde{W} ; \tilde{\sigma}_{\tilde{A}_{n}}(\tilde{\omega})=\tilde{\xi}(\tilde{\omega}) \text { for some } n \text { or } \lim _{n \rightarrow+\infty} \tilde{\sigma}_{\tilde{A}_{n}}(\tilde{\omega})=+\infty\right\},
\end{aligned}
$$

and $\tilde{W}_{1}=\tilde{W}_{11} \cap \tilde{W}_{12}$, then

$$
\tilde{P}_{\tilde{x}}\left(\tilde{W}_{1}\right)=1, \quad \tilde{x} \in \tilde{X} \cup \tilde{\partial}-\tilde{B} .
$$

According to Theorem 3.10, we have a right continuous strong Markov process $\tilde{M}_{1}=\left(\tilde{W}_{1}, \tilde{\mathscr{B}}_{t}^{1}, \tilde{X}_{t}, \tilde{P}_{x}\right)$ with state space $\tilde{X} \cup \tilde{\partial}-\tilde{B}$ which is quasi-left continuous on $[0,+\infty)$.

(II) Definition of $M=\left(\Omega, \mathscr{M}, \mathscr{M}_{t}, X_{t}, P_{x}\right)$. Let us define a set $B \subset X$ by

$$
X-B=q^{-1}(\tilde{X}-\tilde{B}) \text {. }
$$

Since $B=\left(\bigcap_{k=1}^{\infty} A_{k}\right) \cup q^{-1}\left(\tilde{B}-\bigcap_{k=1}^{\infty} \tilde{A}_{k}\right), B$ is a Borel polar set including the set $\bigcap_{k=1}^{\infty} A_{k}$. We put

$$
\Omega=\tilde{W}_{1}, \quad \mathscr{M}=\tilde{\mathscr{B}}^{1}, \quad \mathscr{M}_{t}=\tilde{\mathscr{B}}_{t}^{1} .
$$

The element of $\Omega($ resp. $\mathscr{M})$ is denoted by $\omega($ resp. $\Lambda)$ instead of $\tilde{\omega}$ (resp. $\tilde{\Lambda})$. Define $X_{t}$ and $P_{x}$ by

$$
\begin{array}{ll}
X_{t}(\omega)=q^{-1}\left(\tilde{X}_{t}(\omega)\right), & \omega \in \Omega, t \geqq 0, \\
P_{x}(\Lambda)=\tilde{P}_{q x}(\Lambda), & x \in X \cup \partial-B, \Lambda \in \mathscr{M} .
\end{array}
$$


$X_{t}$ takes values in $X \cup \partial-B$. The field $\mathscr{M}_{t}^{0}=\tilde{\mathscr{B}}_{t}^{0} \cdot \tilde{W}_{1}$ is generated by functions $X_{s}, s \leqq t$, because it is generated by $\tilde{X}_{s}, s \leqq t$, and both $q$ and $q^{-1}$ are one-to-one Borel measurable between $X \cup \partial-B$ and $\tilde{X} \cup \tilde{\partial}-\tilde{B}$. The field $\mathscr{M}^{0}=\tilde{B}^{0} \cdot \tilde{W}_{1}$ is of course generated by $X_{s}, s \geqq 0$.

By (4.4), $P_{x}$ is a probability measure for each $x \in X \cup \partial-B . P_{x}(\Lambda)$ is for each $\Lambda \in \mathscr{M}^{0}$ a Borel measurable function of $x \in X \cup \partial-B$ because it is the composition of two Borel functions $q$ and $\tilde{P} \cdot(\Lambda)$.

The field $\mathscr{M}$ is the completion of $\mathscr{M}^{0}$ with respect to the family of measures $\left\{P_{\mu}(\cdot)=\int_{X \cup \partial-B} \mu(d x) P_{x}(\cdot) ; \mu\right.$ is a finite measure on $\left.X \cup \partial-B\right\}$. This follows from Theorem 3.10(ii) and from the following observation: there is a one-to-one correspondence between finite measures on $X \cup \partial-B$ and those on $\tilde{X} \cup \tilde{\partial}-\tilde{B}$ by the relation $\mu(E)=\tilde{\mu}(q(E))$ and in this case we have $P_{\mu}(\Lambda)=\tilde{P}_{\tilde{\mu}}(\Lambda), \Lambda \in \mathscr{M}^{0}$. The field $\mathscr{M}_{t}$ is the completion of $\mathscr{M}_{t}^{0}$ in $\mathscr{M}$ with respect to the same family of measures.

(III) $M$ is a Hunt process on $X \cup \partial-B$. M has namely the following properties (M.a) (M.e).

(M.a) The sample path $X_{t}$ is right continuous for $t \geqq 0$ and has the left limit in $X \cup \partial-B$ for $t>0, P_{x}$-a.e. $(x \in X \cup \partial-B)$. Further $X_{t}=\partial$ for $t \geqq \zeta P_{x}$-a.e., where $\zeta(\omega)=\inf \left\{t \geqq 0 ; X_{t}(\omega)=\partial\right\}$.

(M.b) $P_{x}\left(X_{0}=x\right)=1, x \in X \cup \partial-B$.

(M.c) $\mathscr{M}_{t}=\mathscr{M}_{t+}, t>0 . \mathscr{M}_{t}$ is the completion in the sense of the preceding paragraph of the $\sigma$-field generated by $\left\{X_{s}, t \geqq s \geqq 0\right\}$.

(M.d) Strong Markov property.

(M.e) Quasi-left continuity.

In the present case, the statement (M.a) is valid for all $\omega \in \Omega$ in view of Theorem 3.10(iv) and (4.1). By (4.2) we see $\tilde{X}_{b} \subset \tilde{B}$ and $P_{x}\left(X_{0}=x\right)=\tilde{P}_{q x}\left(\tilde{X}_{0}=q x\right)=1$, $x \in X \cup \partial-B$ yielding (M.b). The second property of (M.c) is evident by the observation of the preceding paragraph. The first is due to the right continuity of $\tilde{\mathscr{B}}_{t}$. (M.d) follows from Theorem 3.10(i). To see this, consider an $\mathscr{M}_{t}$-stopping time $T$ and a set $\Lambda \in \mathscr{M}_{T}$. Then for any $t>0$ and any Borel set $E \subset X \cup \partial-B$,

$$
\begin{aligned}
P_{x}\left(X_{T+t} \in E, \Lambda\right) & =\tilde{P}_{q x}\left(\tilde{X}_{T+t} \in q E, \Lambda\right)=\tilde{E}_{q x}\left(\tilde{P}_{\tilde{X}_{T}}\left(\tilde{X}_{t} \in q E\right), \Lambda\right) \\
& =\tilde{E}_{q x}\left(P_{q^{-1}\left(\tilde{X}_{T}\right)}\left(X_{t} \in E\right), \Lambda\right)=E_{x}\left(P_{X_{T}}\left(X_{t} \in E\right), \Lambda\right) .
\end{aligned}
$$

(M.e) is due to Theorem 3.10(iv): if $\left\{T_{n}\right\}$ is an increasing sequence of $\mathscr{M}_{t^{-}}$ stopping times and if $T=\lim _{n \rightarrow+\infty} T_{n}$, then

$$
\begin{aligned}
P_{x}\left(\lim _{n \rightarrow+\infty} X_{T_{n}}=X_{T}, T<\infty\right) & =\tilde{P}_{q x}\left(\lim _{n \rightarrow+\infty} X_{T_{n}}=X_{T}, T<\infty\right) \\
& =\tilde{P}_{q x}(T<\infty)=P_{x}(T<\infty) .
\end{aligned}
$$

(IV) The resolvent of the process $M$ generates $(\mathscr{F} *, \mathscr{E})$. If we define the resolvent kernel $\left\{R_{\alpha}(x, E), \alpha>0\right\}$ on $x-B$ by

$$
R_{\alpha}(x, E)=E_{x}\left(\int_{0}^{+\infty} e^{-\alpha t} \chi_{E}\left(X_{t}\right) d t\right)
$$


then for any nonnegative universally measurable function $f$ on $X-B$ which belongs to the space $L^{2}(X ; m)$,

$$
R_{\alpha} f \in \mathscr{F} *
$$

and $R_{\alpha} f$ satisfies the equation (0.5).

In order to prove this statement, put $\tilde{f}=\Phi^{*} f$ with $\Phi^{*}$ defined by (2.2): $\Phi^{*} f(\tilde{x})$ $=f\left(q^{-1} \tilde{x}\right), \tilde{x} \in \tilde{X}-\tilde{B} . \tilde{f}$ is then a nonnegative universally measurable function on $\tilde{X}-\tilde{B}$. If we extend $\tilde{f}$ to a function on $\tilde{X}$ by setting $\tilde{f}(\tilde{x})=0, \tilde{x} \in \tilde{B}$, then $\tilde{f}$ becomes universally measurable on $\tilde{X}$. Further by virtue of (q.2) of Theorem $2.1, \tilde{f} \in L^{2}(\tilde{X} ; \tilde{m})$. Hence we can see by Lemma 3.1 that $\widetilde{R} \alpha \tilde{f}$ belongs to $\tilde{F}^{*}$ and satisfies the equation $\mathscr{E}^{\alpha}\left(\tilde{R}_{\alpha} \tilde{f}, \tilde{v}\right)=(\tilde{f}, \tilde{v})_{X}$ for all $\tilde{v} \in \tilde{\mathscr{F}}$. On the other hand we have $\tilde{R}_{\alpha} \tilde{f}(\tilde{x})=\Phi^{*}\left(R_{\alpha} f\right)(\tilde{x})$, $\tilde{x} \in \tilde{X}-\tilde{B}$, because

$$
\begin{aligned}
R_{\alpha} f(x) & =E_{x}\left(\int_{0}^{+\infty} e^{-\alpha t} f\left(X_{t}\right) d t\right) \\
& =\widetilde{E}_{q x}\left(\int_{0}^{+\infty} e^{-\alpha t} \tilde{f}\left(\tilde{X}_{t}\right) d t\right)=\left(\tilde{R}_{\alpha} \tilde{f}\right)(q x), \quad x \in X-B .
\end{aligned}
$$

Now (q.1) of Theorem 2.1 leads us to (4.9) and the equality $\mathscr{E}^{\alpha}\left(R_{\alpha} f, v\right)$ $=\mathscr{E}_{\alpha}\left(\tilde{R}_{\alpha} \tilde{f}, \Phi^{*} v\right)=\left(\tilde{f}, \Phi^{*} v\right)_{\tilde{X}}$ which is equal to $(f, v)_{X}$ for $v \in \mathscr{F}^{*}$ according to the property (q.2).

4.2. Generalizations of theorems of $\$ 3$. All results of $\S 3$ are still valid when the strongly regular $D$-space and the associated Ray process of $\S 3$ are replaced with the regular $D$-space $(X, m, \mathscr{F}, \mathscr{E})$ and its associated Markov process due to Theorem 4.1 respectively.

We consider a Borel polar set $B \subset X$ and a Markov process

$$
M=\left(\Omega, \mathscr{M}, \mathscr{M}_{t}, X_{t}, P_{x}\right)
$$

on $X \cup \partial-B$ which enjoys the properties (III) and (IV) of subsection 4.1( ${ }^{19}$ ).

Observe that in the course of arguments of $\$ 3$ the speciality of the Ray process that its resolvent leaves the space $C(X)$ invariant has been essentially used nowhere except in the proof of Lemma 3.1. Besides we now have the counterpart of Lemma 3.1, namely, property (IV) of 4.1. Thus all the arguments of $\$ 3$ are immediately applicable to the present context to establish following generalizations.

THEOREM 4.2. If a function $u$ is nonnegative universally measurable on $X-B$, belongs to the space $\mathscr{F}$ and has its regularization $\tilde{u}$ on $X-B$, then $\tilde{u}$ is a quasicontinuous modification of $u$. In particular any excessive function on $X-B$ belonging to $\mathscr{F}$ is an element of $\mathscr{F} *$.

This corresponds to Theorem 3.1. The next is a generalization of Lemma 3.4, Theorems 3.7 and 3.8.

${ }^{(19)}$ It is sufficient to assume property (IV) only for $f \in L^{2} \cap C$. 
Theorem 4.3. Let $A$ be an open or a closed subset of X. Put

$$
\mathscr{F}_{X-A}^{*}=\left\{u \in \mathscr{F}^{*} ; u=0 \text { q.e. on } A\right\} \text {. }
$$

(i) The D-space $\left(\mathscr{F}_{X-A}^{*}, \mathscr{E}\right)$ is generated by the resolvent kernel $R_{\alpha}(x, E)$ $=E_{x}\left(\int_{0}^{\sigma_{A}} e^{-\alpha t} \chi_{E}\left(X_{t}\right) d t\right), \alpha>0$, on $X-B$ : for each nonnegative universally measurable function $f$ on $X-B$ belonging to $L^{2}(X ; m)$, the function $R_{\alpha}^{0} f(x)=\int_{X-B} R_{\alpha}^{0}(x, d y) f(y)$, $x \in X-B$, belongs to the space $\mathscr{F}_{X-A}^{*}$ and the equation $\mathscr{E}^{\alpha}\left(R_{\alpha}^{0} f, v\right)=(f, v)_{X}$ holds for every $v \in \mathscr{F}_{X}^{*}-A$.

(ii) Denote by $\mathscr{H}_{\alpha}^{*}$ the orthogonal complement of $\mathscr{F}_{X-A}^{*}$ in the Hilbert space $\left(\mathscr{F}^{*}, \mathscr{E}^{\alpha}\right)$ and define the kernel $H_{\alpha}(x, E)$ on $X-B$ by

$$
H_{\alpha}(x, E)=E_{x}\left(\exp \left(-\alpha \sigma_{A}\right) ; X_{\sigma_{A}} \in E\right) .
$$

Then the relation $P_{\mathscr{H}}^{*} u=H_{\alpha} u$ holds for every $u \in \mathscr{F} \cap C(X)$ where $P \mathscr{H}_{\alpha}^{*}$ stands for the projection on $\mathscr{H}_{\alpha}^{*}$.

(iii) If $A$ is an open set of the class $\mathscr{U}$ or a compact set, then the equality (3.35) holds q.e. on $X$.

The first assertion of the above theorem generalizes Lemma 3.7(ii) of [8]. Finally we give generalized versions of Theorem 3.11, 12 and 13 .

THEOREM 4.4. A function $u \in \mathscr{F}$ is quasi-continuous if and only if $u$ is finely continuous q.e. on $X$ and universally measurable.

This essentially generalizes a theorem of J. Deny and J. Lions [5, Chapitre II, Théorème 3.2] concerning BLD functions and Cartan's fine topology.

THEOREM 4.5. The following statements are equivalent to each other:

(i) A Borel set $A$ is polar.

(ii) (3.49) holds for m-almost all $x \in X$.

(iii) There exists an m-negligible Borel set $C \supset A \cup B$ such that $X-C$ is $M$ invariant.

THEOREM 4.6. The following conditions are mutually equivalent:

(i) $A$ Borel set $A$ is polar if and only if (3.49) is satisfied for every $x \in X-B$.

(ii) $m$ is a reference measure of the process $M$.

(iii) $R_{\alpha}(x, \cdot)$ is absolutely continuous with respect to $m$ for each $x \in X-B$ and $\alpha>0$.

(iv) $m$ is strictly positive on every nonempty finely open set.

The last condition of Theorem 4.6 follows from condition (iii). The converse is also true because of symmetry of $R_{\alpha}$.

\section{REFERENCES}

1. A. Beurling and J. Deny, Dirichlet spaces, Proc. Nat. Acad. Sci. U.S.A. 45 (1959), 208-215. MR 21 \#5098.

2. R. M. Blumenthal and R. K. Getoor, Markov processes and potential theory, Academic Press, New York, 1968. 
3. J. Deny, Théorie de la capacité dans les espaces fonctionnels, Sém. Théorie Potentiel, dirigé par M. Brelot, G. Choquet et J. Deny, 1964/65, Exposé 1, Secrétariat mathématique, Paris, 1965. MR 32 \#7780.

4. - Méthodes Hilbertiennes et théorie du potentiel, Potential Theory Centro Internazionale Matematico Estivo, Edizioni Cremonese, Roma, 1970, pp. 121-201.

5. J. Deny and J. L. Lions, Les espaces du type de Beppo Levi, Ann. Inst. Fourier (Grenoble) 5 (1953/54), 305-370. MR 17, 646.

6. J. L. Doob, Applications to analysis of a topological definition of smallness of a set, Bull Amer. Math. Soc. 72 (1966), 579-600. MR 34 \#3514.

7. E. B. Dynkin, Markov processes, Fizmatgiz, Moscow, 1963; English transl., Die Grundlehren der math. Wissenschaften, Bände 121, 122, Academic Press, New York; SpringerVerlag, Berlin, 1965. MR 33 \#1886; \#1887.

8. M. Fukushima, A construction of reflecting barrier Brownian motions for bounded domains, Osaka J. Math. 4 (1967), 183-215. MR 37 \#6999.

9. —, On Dirichlet spaces and Dirichlet rings, Proc. Japan Acad. 45 (1969), 433-436. MR 40 \#6610.

10. - Regular representations of Dirichlet spaces, Trans. Amer. Math. Soc. 155 (1971), $455-473$.

11. G. A. Hunt, Markov processes and potentials. I, Illinois J. Math. 1 (1957), 44-93. MR 19, 951.

12. K. Ito and H. P. McKean, Jr., Diffusion processes and their sample paths, Die Grundlehren der math. Wissenschaften, Band 125, Academic Press, New York; Springer-Verlag, Berlin, 1965. MR 33 \#8031.

13. H. Kunita and T. Watanabe, Some theorems concerning resolvents over locally compact spaces, Proc. Fifth Berkeley Sympos. Math. Statist. and Probability (Berkeley, Calif., 1965/66), vol. II: Contributions to Probability Theory, part 2, Univ. of California Press, Berkeley, Calif., 1967, pp. 131-164. MR 35 \#4999.

14. L. H. Loomis, An introduction to abstract harmonic analysis, Van Nostrand, Princeton, N. J., 1953. MR 14, 883.

15. P. A. Meyer, Probability and potentials, Ginn, Blaisdell, Waltham, Mass., 1966. MR 34 \#5119.

16. - Processus de Markov, Lecture Notes in Math., no. 26, Springer-Verlag, Berlin and New York, 1967. MR 36 \#2219.

17. M. Nakai, Algebraic criterion on quasiconformal equivalence of Riemann surfaces, Nagoya Math. J. 16 (1960), 157-184. MR 22 \#1669.

18. D. Ray, Resolvents, transition function, and strongly Markovian processes, Ann. of Math. (2) 70 (1959), 43-72. MR 21 \#6027.

Tokyo University of Education, Tokyo, Japan

UNIVERSITY of IllinoIs, Urbana, ILlinoIs 61801

Current Address: Department of the Foundation of Mathematical Sciences, Tokyo University of Education, Tokyo, Japan 